\title{
Kapitza-Dirac effect in the relativistic regime
}

\author{
Sven Ahrens, ${ }^{1}$ Heiko Bauke, ${ }^{1,}$. ${ }^{7}$ Christoph H. Keitel, ${ }^{1}$ and Carsten Müller ${ }^{1,2,{ }^{7}}$ \\ ${ }^{1}$ Max-Planck-Institut für Kernphysik, Saupfercheckweg 1, 69117 Heidelberg, Germany \\ ${ }^{2}$ Institut für Theoretische Physik I, Heinrich-Heine-Universität Düsseldorf, Universitätsstraße 1, 40225 Düsseldorf, Germany
}

(Dated: October 20, 2018)

A relativistic description of the Kapitza-Dirac effect in the so-called Bragg regime with two and three interacting photons is presented by investigating both numerical and perturbative solutions of the Dirac equation in momentum space. We demonstrate that spin-flips can be observed in the two-photon and the three-photon Kapitza-Dirac effect for certain parameters. During the interaction with the laser field the electron's spin is rotated, and we give explicit expressions for the rotation axis and the rotation angle. The off-resonant Kapitza-Dirac effect, that is, when the Bragg condition is not exactly fulfilled, is described by a generalized Rabi theory. We also analyze the in-field quantum dynamics as obtained from the numerical solution of the Dirac equation.

PACS numbers: 03.65.Pm, 42.50.Ct, 42.55.Vc

\section{Introduction}

The diffraction of electrons at a standing wave of light is referred to as the Kapitza-Dirac effect [1,2]. It is the counterpart process of the usual diffraction of light at a material grating. The observation of the Kapitza-Dirac effect seemed to be feasible with the advent of the laser [3, 4] but its experimental realization was refuted shortly after the claim of detection [5. 7]. The first observation of the Kapitza-Dirac effect in the so-called Bragg regime was achieved by employing atoms [8. 9] in 1986 [10] and two years later in the so-called diffraction regime [11]. The realization of the Kapitza-Dirac effect with electrons was achieved in 1988 [12] in the diffraction regime. The scattering of electrons in the Bragg regime was demonstrated in a precise and sophisticated experimental setup in 2001 [13, 14]. Among all experiments this comes closest to the diffraction process as proposed originally by Kapitza and Dirac [1].

The latter experiment was performed at nonrelativistic laser intensities with a laser field parameter [15] $\xi_{0}=e E_{0} /(m c \omega)=$ $5 \times 10^{-6}$ (with $-e$ denoting the electron's charge and $m$ its mass, laser electric field amplitude $E_{0}$ and angular frequency $\omega$, and the speed of light $c$ ), with nonrelativistic electron momenta $|\boldsymbol{p}|=0.04 \mathrm{mc}$, and at nonrelativistic photon energies $\mathcal{E}=5 \times 10^{-6} m c^{2}$, raising the question [13] of how the KapitzaDirac effect might be modified in the relativistic regime. Higher intensities [16-18] and shorter wavelengths [19-21] are indeed available nowadays, demanding a relativistic theory of Kapitza-Dirac scattering within the framework of the Dirac theory which also accounts for the electron's spin degree of freedom. Beside considerations of the Kapitza-Dirac effect with adiabatic pulse turn-on [22], two electrons [23, 24], and perturbative solutions [25. 28], relativistic investigations have been described in [29, 30] based on the Klein-Gordon equation and therefore neglecting the spin. Freimund and Batelaan raised the question of whether the electron spin is affected in

* heiko.bauke@mpi-hd.mpg.de

$\dagger$ mueller@tp1.uni-duesseldorf.de the Kapitza-Dirac effect, but found a vanishingly small spin-flip probability in the investigated parameter regime by simulating the Bargmann-Michel-Telegdi equations [31]. Another derivation which was carried out by Rosenberg [32] solved the Pauli equation perturbatively in a second-quantized field in the diffraction regime and also found only tiny spin effects. The impact of the electron's spin has also been investigated with respect to free-electron motion [33], bound-electron dynamics [34], atomic photoionization [35], and Compton and Mott [36] scattering in strong plane-wave laser fields. In addition collapse and revival spin dynamics has been put forward in strongly laser-driven electrons [37] and notable spin signatures have been found in laser-induced ionization [38]. Our recent publication on the Kapitza-Dirac effect [39] solves the Dirac equation numerically and perturbatively in the Bragg regime and demonstrates pronounced spin dynamics in the KapitzaDirac effect involving three photons. In the present paper, we elaborate our theory in detail and discuss the possibility of spin dynamics for Kapitza-Dirac scattering with only two interacting photons.

This article is organized as follows. In Sec. 2, we specify the Bragg and the diffraction regimes and characterize the interaction in the Bragg regime employing a classical picture. The quantum equations of motions (namely, the Pauli and Dirac equations) are considered in Sec. 3, where it is shown that for the setup of the Kapitza-Dirac effect they take a particular simple form in momentum space. Based on these equations, we study the Kapitza-Dirac effect with two interacting photons in Sec. 4 numerically and analytically and demonstrate the occurrence of spin flips at relativistic electron momenta. We show that the diffraction probability is independent of the spin orientation of the incident electron beam. The spin orientation of the diffracted electrons is rotated as a result of the interaction with the laser field. We also discuss the resonance peak structure of the diffraction within a generalized Rabi theory. In Sec. 5 , we investigate the Kapitza-Dirac effect with three interacting photons in close analogy to the two-photon case. We analyze the in-field quantum dynamics of the Kapitza-Dirac effect and we discuss the tilt of the axis about which the electron spin is rotated. 


\section{Semi-classical considerations}

Depending on the parameters of the laser and the incident electron, the scattering dynamics of the Kapitza-Dirac effect happens in different regimes. Phenomenologically one refers to the Bragg regime if the electron is scattered into a single diffraction order and one refers to the diffraction regime if many diffraction orders can be reached. Employing an argument based on the time-energy uncertainty relation [14, 40] one can show that if the interaction time of the electron with the laser is short, the electron is diffracted into several diffraction orders. Long interaction times, however, permit dynamics in the Bragg regime [25]. In the Bragg regime the electron can be diffracted only if it fulfills the classical energy and momentum conservation. This article focuses on the Kapitza-Dirac effect in the Bragg regime.

The Kapitza-Dirac effect can be viewed as a scattering process where the electron either absorbs or emits photons from or into the counter-propagating laser beams. We denote the number of absorbed photons from the left- (right-) traveling laser beam by $n_{l}\left(n_{r}\right)$. Negative values of $n_{l}$ or $n_{r}$ correspond to photon emission of the electron into the left or the right laser beam. Following this notion, classical momentum conservation requires

$$
\boldsymbol{p}_{\text {out }}=\boldsymbol{p}_{\text {in }}+n_{r} \boldsymbol{k}-n_{l} \boldsymbol{k} .
$$

Note that Gaussian units are used in this article and we set $\hbar=1$. Furthermore, energy conservation implies

$$
\mathcal{E}\left(\boldsymbol{p}_{\text {out }}\right)=\mathcal{E}\left(\boldsymbol{p}_{\text {in }}\right)+n_{r} c k+n_{l} c k,
$$

with $\boldsymbol{p}_{\text {in }}$ and $\boldsymbol{p}_{\text {out }}$ denoting the electron's momentum before and after interaction with the laser, the electron's kinetic energy $\mathcal{E}(\boldsymbol{p})$, and the photon momentum $\boldsymbol{k}$. For convenience, we separate the electron's momentum into components in the laser propagation direction $p_{k}$, the laser polarization direction $p_{E}$, and the direction of the laser magnetic field $p_{B}$ (see Fig. 1). Applying the nonrelativistic energy-momentum relation

$$
\mathcal{E}^{\mathrm{nr}}(\boldsymbol{p})=\frac{\boldsymbol{p}^{2}}{2 m}
$$

the solution of Eq. (2) with respect to $p_{k \text {,in }}$ yields

$$
p_{k, \text { in }}^{\mathrm{nr}}=-\frac{n_{r}-n_{l}}{2} k+\frac{n_{r}+n_{l}}{n_{r}-n_{l}} m c,
$$

whereas Eq. (2) with the relativistic energy-momentum relation

$$
\mathcal{E}^{\mathrm{r}}(\boldsymbol{p})=\sqrt{m^{2} c^{4}+c^{2} \boldsymbol{p}^{2}}
$$

yields

$$
\begin{aligned}
& p_{k, \text { in }}^{\mathrm{r}}=-\frac{n_{r}-n_{l}}{2} k \\
& \quad+\frac{n_{r}-n_{l}}{\left|n_{r}-n_{l}\right|} \frac{n_{r}+n_{l}}{2} \sqrt{k^{2}-\frac{m^{2} c^{2}+\left(p_{B, \mathrm{in}}^{\mathrm{r}}\right)^{2}+\left(p_{E, \mathrm{in}}^{\mathrm{r}}\right)^{2}}{n_{r} n_{l}}} .
\end{aligned}
$$

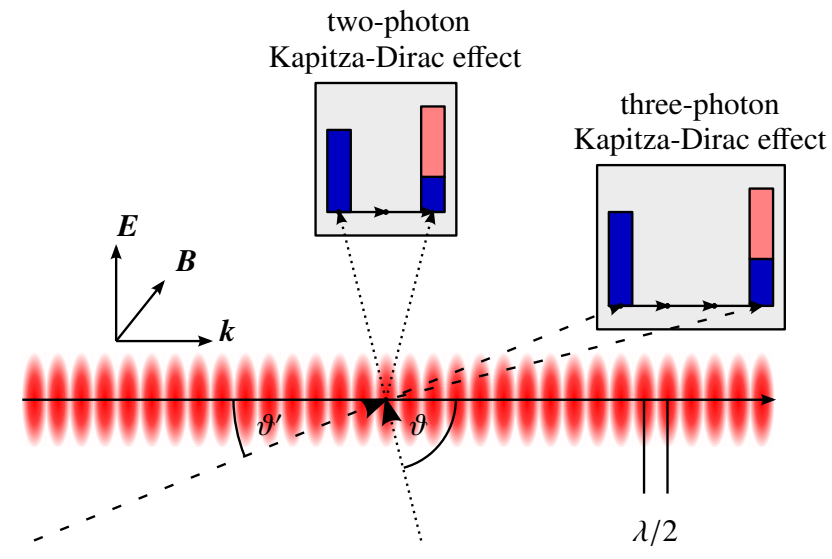

Figure 1: (Color online) Setup of the two-photon and the threephoton Kapitza-Dirac effects. If the electron is diffracted, it may pick up two photon momenta or three photon momenta, respectively, from the laser field. Directions of the electron beams are indicated by dotted (two-photon Kapitza-Dirac effect) and dashed (three-photon Kapitza-Dirac effect) lines. The gray panels emblematize screens, which display the intensity of the scattered and unscattered electron beams (colored bars), depending on the electron momentum in the laser propagation direction. In both Kapitza-Dirac effects spin flips may occur, indicated by the light red bar of the diffraction pattern as compared to the dark blue bars of the unflipped electron beam intensity. According to the considerations of Secs. 2 and 4 the electron enters the laser almost perpendicular to the laser's propagation direction for the two-photon Kapitza-Dirac effect with spin-flip dynamics, implying $\vartheta \approx 90^{\circ}$. For the three-photon Kapitza-Dirac effect, however, the energy-momentum conservation constraint (6) results in relativistic momenta of the electron in the laser propagation direction such that typically $\vartheta^{\prime} \ll 90^{\circ}$.

Note that the solution (6) is real-valued and finite only if $n_{r}$ and $n_{l}$ are non-zero and have opposite signs. This means that the Kapitza-Dirac effect in the Bragg regime requires that at least one photon is absorbed from one laser beam and at least one photon is emitted into the counterpropagating laser beam. For $n_{r}+n_{l}=0$ the electron's energy (2) is conserved and, therefore, the scattering is elastic; otherwise it is inelastic.

Equation (4) as well as Eq. (6) yield $p_{k, \text { out }}^{\mathrm{nr} / \mathrm{r}}=-p_{k, \text { in }}^{\mathrm{nr} / \mathrm{r}}$ for elastic electron scattering corresponding to "The reflection of electrons from standing light waves" proposed by Kapitza and Dirac [1]. Energy-momentum conservation can be illustrated in an energy-momentum diagram as shown in Fig. 2 (a) for the elastic two-photon Kapitza-Dirac effect.

In the case of the three-photon Kapitza-Dirac effect, two laser photons are absorbed $\left(n_{r}=2\right)$ and one is emitted $\left(n_{l}=-1\right)$. The energy-momentum diagram of this process is sketched in Fig. 2(b). Processes with $n_{r}=-1$ and $n_{l}=2$, $n_{r}=-2$ and $n_{l}=1$, or $n_{r}=1$ and $n_{l}=-2$ are also possible but not fundamentally different from the Kapitza-Dirac effect with $n_{r}=2$ and $n_{l}=-1$ and therefore not considered here. For such an inelastic process, energy is transferred from the laser field to the electron. This energy transfer is indicated by a dashed line in Fig. 2(b). Approaching the limit $\boldsymbol{k} \rightarrow 0$, the dashed line in Fig. 2(b) is shifted downwards while preserving its slope until it becomes a tangent of the energy hyperbola 

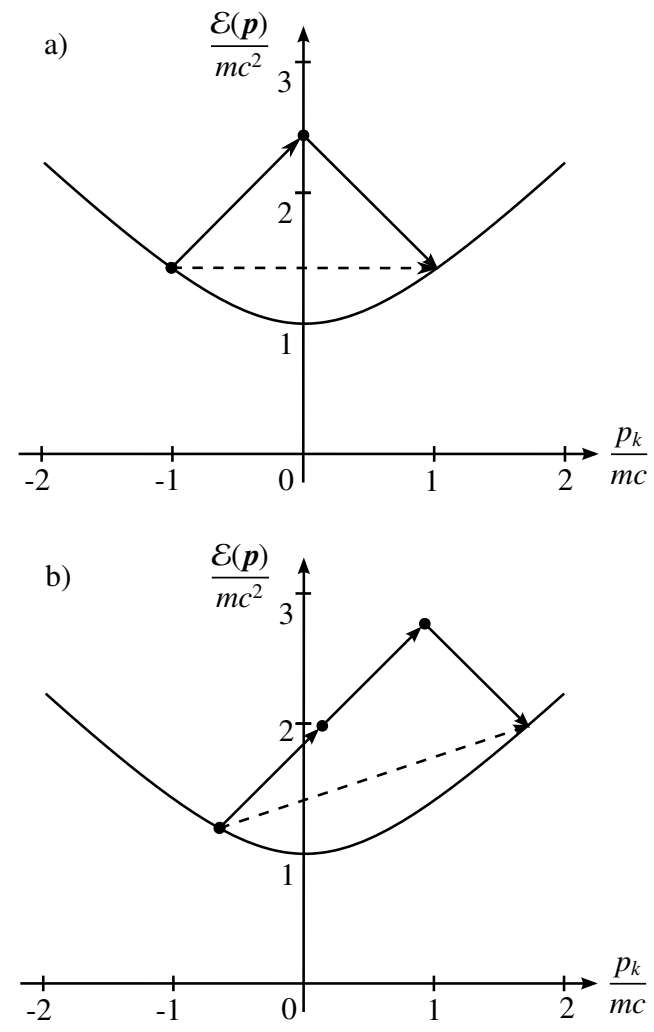

Figure 2: Sketch of the energy and momentum conservation in the Kapitza-Dirac effect. The electron must reside on its relativistic energy-momentum relation (5) (solid hyperbola) before as well as after the interaction, and the laser can transfer only energy and momentum quanta which fulfill $\Delta \mathcal{E}=c k$ (diagonal arrows). In the two-photon Kapitza-Dirac effect (a), one photon is absorbed and one photon is emitted, whereas in the three-photon Kapitza-Dirac effect (b) two photons are absorbed and one photon is emitted. In the latter process the energy of the electron changes during diffraction as indicated by the nonhorizontal dashed line.

(5). Consequently, energy-momentum conservation requires a non-vanishing initial momentum of the electron in the laser propagation direction even for small photon momenta. Furthermore, the energy conservation (2) enforces that at least one of the momenta $k, p_{k}, p_{E}$, or $p_{B}$ is relativistic, i. e., of the order of or larger than $m c$. Consequently, no nonrelativistic limit of (6) exists in this case. For example, the momenta (4) and (6) do not converge even for small laser photon momenta $k$. Rather we find with $n_{r}=2$ and $n_{l}=-1$ the differing limiting values

$$
\lim _{k \rightarrow 0} p_{k, \text { in }}^{\mathrm{nr}}=\frac{m c}{3}
$$

and

$$
\lim _{k \rightarrow 0} p_{k, \text { in }}^{\mathrm{r}}=\sqrt{\frac{m^{2} c^{2}+p_{E}^{2}+p_{B}^{2}}{8}} .
$$

For $p_{k, \text { in }}^{\mathrm{r}}$ to be small compared to $m c$ the number of interacting photons has to go to infinity such that $\left|n_{r}-n_{l}\right| \gg 1$ and $\left|n_{r}+n_{l}\right| \approx 1$. In this case the slope of the energy-momentum transfer (dashed lines in Fig. 2) goes to zero. Therefore, the touching point of the corresponding tangent that results in the limit $\boldsymbol{k} \rightarrow 0$ lies at small electron momenta in laser propagation direction for Kapitza-Dirac scattering with a high number of interacting photons.

\section{Quantum dynamics in momentum space}

In order to study spin effects and the quantum dynamics of the two-photon and three-photon Kapitza-Dirac effects in a relativistic setting we will solve the time-dependent Dirac equation. For two-photon interactions we will also utilize the nonrelativistic Pauli equation given that relativistic parameters are not mandatory in this case. As we will show in this section, the Pauli equation and the Dirac equation for the setup of the Kapitza-Dirac effect can be reduced to a system of ordinary differential equations by transforming them into momentum space

\subsection{Laser setup}

For the Kapitza-Dirac effect we consider two counterpropagating linearly polarized lasers of equal intensity and angular frequency $\omega$. The vector potential of this laser setup is given by

$$
\boldsymbol{A}(\boldsymbol{x}, t)=\hat{\boldsymbol{A}}(t) \cos (\boldsymbol{k} \cdot \boldsymbol{x}) \sin (\omega t),
$$

where we have introduced the temporal envelope function

$$
\hat{\boldsymbol{A}}(t)=\hat{\boldsymbol{A}}_{\max } \times \begin{cases}\sin ^{2} \frac{\pi t}{2 \Delta T} & \text { if } 0 \leq t \leq \Delta T \\ 1 & \text { if } \Delta T \leq t \leq T-\Delta T \\ \sin ^{2} \frac{\pi(T-t)}{2 \Delta T} & \text { if } T-\Delta T \leq t \leq T, \\ 0 & \text { else, }\end{cases}
$$

which allows for a smooth turn-on and turn-off of the laser field. The variables $T$ and $\Delta T$ denote the total interaction time and the time of turn-on and turn-off. After turn-on and before turn-off, the electric and magnetic amplitudes of the oscillating electromagnetic fields are given by

$$
\begin{aligned}
& \hat{\boldsymbol{E}}=k \hat{\boldsymbol{A}}_{\max }, \\
& \hat{\boldsymbol{B}}=-\boldsymbol{k} \times \hat{\boldsymbol{A}}_{\max } .
\end{aligned}
$$

For convenience, we will choose our coordinate system such that the orthogonal vectors $\boldsymbol{k}, \hat{\boldsymbol{B}}$, and $\hat{\boldsymbol{E}}$ point along the $x, y$, and $z$ directions. In the following all vector quantities will be projected in the directions of $\boldsymbol{k}, \hat{\boldsymbol{E}}$, and $\hat{\boldsymbol{B}}$ as indicated by the indices $k, E$, and $B$, respectively. For simplicity, we omit the index $E$ for the vector potential, because the vector potential always points in the electric field direction, that is, $\hat{A}(t)=\hat{A}_{E}(t)$. 


\subsection{The Pauli equation}

The Pauli equation that governs the quantum motion of a spin$1 / 2$ particle of mass $m$ and charge $q$ is given by

$\mathrm{i} \dot{\psi}(\boldsymbol{x}, t)=\frac{1}{2 m}\left(-\mathrm{i} \nabla-\frac{q}{c} \boldsymbol{A}(\boldsymbol{x}, t)\right)^{2} \psi(\boldsymbol{x}, t)-\frac{q}{2 m c} \boldsymbol{\sigma} \cdot \boldsymbol{B}(\boldsymbol{x}, t) \psi(\boldsymbol{x}, t)$

with the Pauli matrices $\sigma=\left(\sigma_{1}, \sigma_{2}, \sigma_{3}\right)^{\top}=\left(\sigma_{k}, \sigma_{B}, \sigma_{E}\right)^{\top}$ and $\boldsymbol{B}(\boldsymbol{x}, t)=\nabla \times \boldsymbol{A}(\boldsymbol{x}, t)$. Taking advantage of the sinusoidal spatial periodicity of the vector potential $\boldsymbol{A}(\boldsymbol{x}, t)$, we expand the wave function

$$
\psi(\boldsymbol{x}, t)=\sum_{n, \zeta} c_{n}^{\zeta}(t) \psi_{n}^{\zeta}(\boldsymbol{x})
$$

into momentum eigenfunctions of the free Pauli Hamiltonian

$$
\psi_{n}^{\zeta}(\boldsymbol{x})=\sqrt{\frac{k}{2 \pi}} \chi^{\zeta} \mathrm{e}^{\mathrm{i} \boldsymbol{p}_{n} \cdot \boldsymbol{x}}
$$

with $\zeta \in\{\uparrow, \downarrow\}$, the two spinor basis functions

$$
\chi^{\uparrow}=\left(\begin{array}{l}
1 \\
0
\end{array}\right), \quad \quad \chi^{\downarrow}=\left(\begin{array}{l}
0 \\
1
\end{array}\right),
$$

and the momentum $\boldsymbol{p}_{n}=\boldsymbol{p}+n \boldsymbol{k}$. Inserting the ansatz 13. into the Pauli equation (12) and projecting onto the basis elements (14) from the left-hand side yields the Pauli equation in momentum space

$$
\begin{aligned}
& \mathrm{i} \dot{c}_{n}(t)= \\
& \frac{(\boldsymbol{p}+n \boldsymbol{k})^{2}}{2 m} c_{n}(t)+\frac{q^{2} \hat{A}(t)^{2}}{8 m c^{2}} \sin ^{2}(\omega t)\left[c_{n-2}(t)+2 c_{n}(t)+c_{n+2}(t)\right] \\
& -\frac{q \sin (\omega t)}{4 m c} \sum_{\delta n \in\{1,-1\}}\left[2 \hat{A}(t) p_{E} \mathbb{1}-\mathrm{i} \delta n \hat{\boldsymbol{k}} \times \hat{\boldsymbol{A}}(t) \sigma_{B}\right] c_{n+\delta n}(t),
\end{aligned}
$$

where the vectors $c_{n}(t)=\left(c_{n}^{\uparrow}(t), c_{n}^{\downarrow}(t)\right)^{\top}$ and the $2 \times 2$ identity matrix $\mathbb{1}$ have been introduced.

\subsection{The Dirac equation}

The Dirac equation is a relativistic generalization of the nonrelativistic Pauli equation. It is given by

$$
\mathrm{i} \dot{\psi}(\boldsymbol{x}, t)=c \boldsymbol{\alpha} \cdot\left(-\mathrm{i} \nabla-\frac{q}{c} \boldsymbol{A}(\boldsymbol{x}, t)\right) \psi(\boldsymbol{x}, t)+m c^{2} \beta \psi(\boldsymbol{x}, t)
$$

with $\alpha=\left(\alpha_{1}, \alpha_{2}, \alpha_{3}\right)^{\top}$ and $\beta$ denoting the Dirac matrices [41]. We transform the Dirac equation (17) into momentum space in close analogy to the momentum space transformation of the Pauli equation (16). For this purpose, the basis elements (14) of the wave function 13 are replaced by

$$
\psi_{n}^{\gamma}(\boldsymbol{x})=\sqrt{\frac{k}{2 \pi}} u_{n}^{\gamma} \mathrm{e}^{\mathrm{i} \boldsymbol{p}_{n} \cdot \boldsymbol{x}}
$$

The bi-spinors $u_{n}^{\gamma}$ with $\gamma \in\{+\uparrow,-\uparrow,+\downarrow,-\downarrow\}$ are explicitly given by

$$
u_{n}^{+\zeta}=\sqrt{\frac{\mathcal{E}_{n}+m c^{2}}{2 \mathcal{E}_{n}}}\left(\begin{array}{c}
\chi^{\zeta} \\
\frac{\sigma \cdot c p_{n}}{\mathcal{E}_{n}+m c^{2}}
\end{array} \chi^{\zeta}\right)
$$

and

$$
u_{n}^{-\zeta}=\sqrt{\frac{\mathcal{E}_{n}+m c^{2}}{2 \mathcal{E}_{n}}}\left(\begin{array}{c}
-\frac{\boldsymbol{\sigma} \cdot c \boldsymbol{p}_{n}}{\mathcal{E}_{n}+m c^{2}} \chi^{\zeta} \\
\chi^{\zeta}
\end{array}\right) .
$$

The basis functions $\psi_{n}^{ \pm \zeta}(\boldsymbol{x})$ are simultaneously eigenfunctions of the free time-independent Dirac equation with energy eigenvalue $\pm \mathcal{E}_{n}$ defined as

$$
\mathcal{E}_{n}=\sqrt{m c^{2}+c^{2} \boldsymbol{p}_{n}^{2}}
$$

and eigenfunctions of the momentum operator with momentum eigenvalue $\boldsymbol{p}_{n}$. Furthermore, $\psi_{n}^{ \pm \uparrow}(\boldsymbol{x})$ and $\psi_{n}^{ \pm \downarrow}(\boldsymbol{x})$ are eigenfunctions of the Foldy-Wouthuysen spin operator along the $\hat{\boldsymbol{E}}$ direction with eigenvalues $1 / 2$ and $-1 / 2$ [42-45].

Inserting the ansatz (13) with the basis elements (19) in the Dirac equation 12 and projecting with these from the left-hand side yields the Dirac equation in momentum space

$$
\mathrm{i} \dot{c}_{n}(t)=\mathcal{E}_{n} \beta c_{n}(t)-\frac{q \sin (\omega t)}{2}\left(L_{n, n-1} c_{n-1}(t)+L_{n, n+1} c_{n+1}(t)\right),
$$

with the vectors

$$
c_{n}(t)=\left(c_{n}^{+\uparrow}(t), c_{n}^{+\downarrow}(t), c_{n}^{-\uparrow}(t), c_{n}^{-\downarrow}(t)\right)^{\top}
$$

and the coupling matrices $L_{n, n^{\prime}}$ whose elements are defined as

$$
L_{n, n^{\prime}}^{\gamma, \gamma^{\prime}}=u_{n}^{\gamma \dagger}(\boldsymbol{\alpha} \cdot \hat{\boldsymbol{A}}(t)) u_{n^{\prime}}^{\gamma^{\prime}}
$$

\subsection{Numerical procedure}

The absolute square values of the expansion coefficients $c_{n}^{\zeta}(t)$ in (13) represent the probability of finding the electron in a particular free-particle quantum state. The state that is represented by $c_{n}^{\zeta}(t)$ has definite spin that is encoded in the index $\zeta$ and also has a definite momentum $\boldsymbol{p}_{n}=\boldsymbol{p}+n \boldsymbol{k}$. Thus, the index $n$ counts the number of laser photon momenta relative to the reference momentum $\boldsymbol{p}$. For convenience, we use the term "mode $n$ " for these different electron momenta.

In numerical simulations, we start from an initial quantum state with definite momentum $\boldsymbol{p}$ and spin-up polarization. This means

$$
c_{n}^{\gamma}(0)= \begin{cases}1 & \text { if } n=0 \text { and } \gamma=+\uparrow \text { or } \gamma=\uparrow \\ 0 & \text { else. }\end{cases}
$$

The case $\gamma=+\uparrow$ applies for the Dirac equation, while $\gamma=\uparrow$ is for the Pauli equation. The initial electron momentum $\boldsymbol{p}$, the laser intensity, and the laser frequency are chosen to meet the 


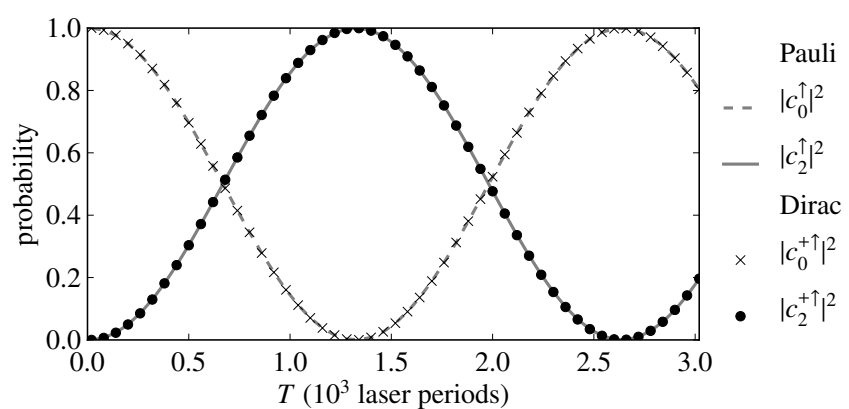

Figure 3: Occupation probabilities as a function of the total interaction time $T$ for the two-photon Kapitza-Dirac effect calculated by employing the Dirac equation (crosses and dots) 21) and the Pauli equation (16) (solid and dashed lines). The electron enters the laser field with a momentum component of $p_{E}=-12.5 \mathrm{keV} / c=2.4 \times 10^{-2} \mathrm{mc}$ along the laser electric field direction and interacts with the standing laser field of peak intensity $5 \times 10^{21} \mathrm{~W} / \mathrm{cm}^{2}$ (corresponding to $e \hat{A}_{\max }=8.6 \times 10^{-3} m c^{2}$ ) for each laser beam and a photon energy of $12.5 \mathrm{keV}$ (corresponding to a laser wave length of $\lambda=0.1 \mathrm{~nm}$ ). The probability that the electron is diffracted from mode 0 to mode 2 oscillates in Rabi cycles as a function of the total interaction time $T$. The laser field is modulated via the envelope function 9 with a turn-on and turn-off time of ten laser periods.

nonrelativistic Bragg condition (4) or its relativistic generalization (6) depending on whether the Pauli equation or the Dirac equation is solved numerically. The numerical solution of the differential equations (16) and (21) is obtained by employing a Crank-Nicholson scheme [46]. Equations (16) and (21) couple an infinite number of modes. In numerical simulations, however, these systems are truncated to a finite number of modes, $-n_{\max } \leq n \leq n_{\max }$ with $n_{\max }$ large enough such that the physical results are independent of $n_{\max }$. The number of included modes depends on the laser parameters and ranges typically from one dozen to several dozens. The duration of the turn-on and turn-off phases $\Delta T$ is ten laser periods for all simulations presented in this article unless another turn-on and turn-off time is indicated.

Note that our approach to solving the Dirac equation is tailored for interactions with monochromatic laser fields. More general approaches include solving the Dirac equation via a Fourier transform split-operator method [47], in particular by employing geometric algebra [48] and by making use of a graphics processing unit [49]. Other methods employ spherical harmonics as basis functions and Runge-Kutta integration [50] or are based on the method of characteristics [51].

\section{Two-photon Kapitza-Dirac effect}

\subsection{Numerical results}

In the electron scattering dynamics as described by Kapitza and Dirac [1] one photon is absorbed from the laser field and one is emitted into the laser field, $n_{r}=1$ and $n_{l}=-1$ in our notation. For realizing this effect the parameters of the laser as well as of the incident electron have to be chosen such that the quantum dynamics is in the Bragg regime [40]. For $n_{r}+n_{l}=0$ the nonrelativistic Bragg condition (4) as well as the relativistic Bragg condition (6) require that the electron enters the laser beam with $p_{k}=-k$. For the numerical simulations shown in Fig. 3 we choose $\boldsymbol{p}=-k \boldsymbol{e}_{k}+k \boldsymbol{e}_{E}$ with $k=2.4 \times 10^{-2} m c$ and $e \hat{A}_{\max }=8.6 \times 10^{-3} m c^{2}$.

Figure 3 shows the quantum state after turn-off of the laser for the two-photon Kapitza-Dirac effect for different total interaction times $T$ as calculated by solving the Dirac and the Pauli equation numerically. As all parameters are in the nonrelativistic regime the Dirac equation and the Pauli equation give qualitatively and quantitatively the same results. The quantum dynamics exhibits the well-known Rabi oscillations of the diffraction probability [40] from mode 0 with momentum $\boldsymbol{p}=-k \boldsymbol{e}_{k}+k \boldsymbol{e}_{E}$ to mode 2 with momentum $\boldsymbol{p}=+k \boldsymbol{e}_{k}+k \boldsymbol{e}_{E}$, in the form

$$
\begin{aligned}
\left|c_{0}^{+\uparrow}(T)\right|^{2} & =\cos ^{2}\left(\frac{\Omega_{R} T}{2}\right), \\
\left|c_{2}^{+\uparrow}(T)\right|^{2} & =\sin ^{2}\left(\frac{\Omega_{R} T}{2}\right) .
\end{aligned}
$$

Here, $\Omega_{R}$ denotes the Rabi frequency. The occupation probability of all other modes is vanishingly small, in particular, $\left|c_{2}^{+\downarrow}(T)\right|^{2}<10^{-6}$. This means that no spin flip occurs during two-photon Kapitza-Dirac scattering. From a naive point of view the vanishing spin-flip probability might be surprising, because one might expect a precession of the electron spin in the magnetic field of the external laser field. The question of electron spin precession has already been investigated based on nonrelativistic classical equations of motion [31] but no significant spin effects could be found. In Sec. 4.4, however, we will show that spin-flips are possible in the two-photon Kapitza-Dirac effect for certain relativistic parameter settings.

\subsection{Perturbation theory}

In the following we will complement our numerical findings with analytical results obtained via time-dependent perturbation theory. Time-dependent perturbation theory for the Dirac equation 21] will allow us to calculate analytical expressions for the Rabi frequency and to derive conditions that permit spin-flip dynamics in the two-photon Kapitza-Dirac effect. The perturbative solution also allows for deduction of a rotation of the electron spin during diffraction. As the initial condition is given by 24) and the electron momentum is changed by two photon momenta our aim is to approximate the time evolution operator $U_{2,0}(t, 0)$ that maps $c_{0}(0)$ to $c_{2}(t)$, viz.,

$$
c_{2}(t)=U_{2,0}(t, 0) c_{0}(0) \text {. }
$$

Since the Dirac equation (21) couples next neighboring modes only, the lowest non-vanishing contribution to $U_{2,0}(t, 0)$ is of second order in time-dependent perturbation theory. The 
general second order propagator for a time-dependent Hamiltonian $H(t)$ reads [52]

$$
\begin{aligned}
& U_{\mathrm{nd}}(t, 0)= \\
& \frac{1}{\mathrm{i}^{2}} \int_{0}^{t} \mathrm{~d} t_{2} \int_{0}^{t_{2}} \mathrm{~d} t_{1} U_{0}\left(t, t_{2}\right) V\left(t_{2}\right) U_{0}\left(t_{2}, t_{1}\right) V\left(t_{1}\right) U_{0}\left(t_{1}, 0\right) .
\end{aligned}
$$

The symbol $U_{0}(t, 0)$ denotes the free propagator $U_{0}(t, 0)=$ $\mathrm{e}^{-\mathrm{i} H_{0} t}$, with the time-independent field-free Hamiltonian

$$
H_{0}=\left(\begin{array}{lllll}
\ddots & & & & \\
& H_{0 ; 1,1} & & & \\
& & H_{0 ; 0,0} & & \\
& & & H_{0 ;-1,-1} & \\
& & & & \ddots
\end{array}\right)
$$

with $H_{0 ; a, b}=\mathcal{E}_{a} \beta \delta_{a, b}$ and the time-dependent interaction Hamiltonian $V(t)=H(t)-H_{0}$. For the Dirac equation in momentum space 21, the free propagator reads explicitly

$$
U_{0 ; a, b}(t, 0)=\mathrm{e}^{-\mathrm{i} \mathcal{E}_{a} \beta t} \delta_{a, b}=\left(\begin{array}{cc}
\exp \left(-\mathrm{i} \mathcal{E}_{a} t\right) \mathbb{1} & 0 \\
0 & \exp \left(\mathrm{i} \mathcal{E}_{a} t\right) \mathbb{1}
\end{array}\right) \delta_{a, b}
$$

The corresponding interaction Hamiltonian reads

$$
V_{a, b}(t)=-\frac{q \sin (\omega t)}{2}\left(L_{a, a-1} \delta_{a, b+1}+L_{a, a+1} \delta_{a, b-1}\right)
$$

Inserting these expressions into 27] yields

$$
\begin{aligned}
U_{\mathrm{nd} ; 2,0}(t, 0)=- & \int_{0}^{t} \mathrm{~d} t_{2} \int_{0}^{t_{2}} \mathrm{~d} t_{1} \mathrm{e}^{-\mathrm{i} \varepsilon_{2} \beta\left(t-t_{2}\right)} \frac{q \sin \left(\omega t_{2}\right)}{2} L_{2,1} \\
& \times \mathrm{e}^{-\mathrm{i} \mathcal{E}_{1} \beta\left(t_{2}-t_{1}\right)} \frac{q \sin \left(\omega t_{1}\right)}{2} L_{1,0} \mathrm{e}^{-\mathrm{i} \mathcal{E}_{0} \beta t_{1}}
\end{aligned}
$$

In order to ease notations, it will be useful to split the $4 \times 4$ matrices $L_{n, n^{\prime}}$ and $U_{\mathrm{nd} ; 2,0}(t, 0)$ into blocks of $2 \times 2$ matrices, viz.,

$$
L_{n, n^{\prime}}=\left(\begin{array}{cc}
L_{n, n^{\prime}}^{++} & L_{n, n^{\prime}}^{+-} \\
L_{n, n^{\prime}}^{-+} & L_{n, n^{\prime}}^{--}
\end{array}\right) \quad \text { with } \quad L_{n, n^{\prime}}^{a b}=\left(\begin{array}{cc}
L_{n, n^{\prime}}^{a \uparrow, b \uparrow} & L_{n, n^{\prime}}^{a \uparrow, b \downarrow} \\
L_{n, n^{\prime}}^{a \downarrow, b \uparrow} & L_{n, n^{\prime}}^{a \downarrow, b \downarrow}
\end{array}\right)
$$

and

$$
U_{\mathrm{nd} ; 2,0}(t, 0)=\left(\begin{array}{cc}
U_{\mathrm{nd} ; 2,0}^{++}(t, 0) & U_{\mathrm{nd} ; 2,0}^{+-}(t, 0) \\
U_{\mathrm{nd} ; 2,0}^{-+}(t, 0) & U_{\mathrm{nd} ; 2,0}^{--}(t, 0)
\end{array}\right)
$$

Explicit expressions for the matrices $L_{n, n^{\prime}}^{a b}$ are given in the Appendix A With these definitions the sub-propagator $U_{\mathrm{nd} ; 2,0}^{++}(t, 0)$ in the space of positive-energy free-particle states reads for times after the turn-on phase and before the turn-off phase $(\Delta T=0$ and $0<t<T)$

$$
U_{\mathrm{nd} ; 2,0}^{++}(t, 0)=-\frac{q^{2}}{4} L_{2,1}^{++} L_{1,0}^{++} \int_{0}^{t} \mathrm{~d} t_{2} \int_{0}^{t_{2}} \mathrm{~d} t_{1} \sin \left(\omega t_{2}\right) \sin \left(\omega t_{1}\right) v^{+}\left(t, t_{2}, t_{1}\right)-\frac{q^{2}}{4} L_{2,1}^{+-} L_{1,0}^{-+} \int_{0}^{t} \mathrm{~d} t_{2} \int_{0}^{t_{2}} \mathrm{~d} t_{1} \sin \left(\omega t_{2}\right) \sin \left(\omega t_{1}\right) v^{-}\left(t, t_{2}, t_{1}\right),
$$

where we have introduced the two complex phases

$$
\begin{aligned}
& v^{+}\left(t, t_{2}, t_{1}\right)=\exp \left[-\mathrm{i}\left(\mathcal{E}_{2} t+\Delta \mathcal{E}_{1,2}^{++} t_{2}+\Delta \mathcal{E}_{0,1}^{++} t_{1}\right)\right], \\
& v^{-}\left(t, t_{2}, t_{1}\right)=\exp \left[-\mathrm{i}\left(\mathcal{E}_{2} t+\Delta \mathcal{E}_{1,2}^{-+} t_{2}+\Delta \mathcal{E}_{0,1}^{+-} t_{1}\right)\right] .
\end{aligned}
$$

Here, $\Delta \mathcal{E}_{n, n^{\prime}}^{a b}$ is an abbreviation for the energy difference $\Delta \mathcal{E}_{n, n^{\prime}}^{a b}=\operatorname{sign}(a) \mathcal{E}_{n}-\operatorname{sign}(b) \mathcal{E}_{n^{\prime}}$, where the signum of the upper indices is $\operatorname{sign}(+)=1$ and $\operatorname{sign}(-)=-1$. Performing the first integral in 34 we find

$$
\begin{aligned}
& \int_{0}^{t} \mathrm{~d} t_{2} \int_{0}^{t_{2}} \mathrm{~d} t_{1} \sin \left(\omega t_{2}\right) \sin \left(\omega t_{1}\right) v^{+}\left(t, t_{2}, t_{1}\right)= \\
&-\frac{1}{4} \mathrm{e}^{-\mathrm{i} \mathcal{E}_{2} t} \int_{0}^{t} \mathrm{~d} t_{2}\left[\frac{\mathrm{i}}{\Delta \mathcal{E}_{0,1}^{++}+\omega}\left(\mathrm{e}^{-\mathrm{i}\left(\Delta \mathcal{E}_{0,2}^{++}+2 \omega\right) t_{2}}-\mathrm{e}^{-\mathrm{i}\left(\Delta \mathcal{E}_{1,2}^{++}+\omega\right) t_{2}}\right)-\frac{\mathrm{i}}{\Delta \mathcal{E}_{0,1}^{++}-\omega}\left(\mathrm{e}^{-\mathrm{i} \Delta \mathcal{E}_{0,2}^{++} t_{2}}-\mathrm{e}^{-\mathrm{i}\left(\Delta \mathcal{E}_{1,2}^{++}+\omega\right) t_{2}}\right)\right. \\
&\left.\quad-\frac{\mathrm{i}}{\Delta \mathcal{E}_{0,1}^{++}+\omega}\left(\mathrm{e}^{-\mathrm{i} \Delta \mathcal{E}_{0,2}^{++} t_{2}}-\mathrm{e}^{-\mathrm{i}\left(\Delta \mathcal{E}_{1,2}^{++}-\omega\right) t_{2}}\right)+\frac{\mathrm{i}}{\Delta \mathcal{E}_{0,1}^{++}-\omega}\left(\mathrm{e}^{-\mathrm{i}\left(\Delta \mathcal{E}_{0,2}^{++}-2 \omega\right) t_{2}}-\mathrm{e}^{-\mathrm{i}\left(\Delta \mathcal{E}_{1,2}^{++}-\omega\right) t_{2}}\right)\right] .
\end{aligned}
$$

The integral (37) may show linear or oscillating behavior depending on the laser parameters and the initial electron momentum $\boldsymbol{p}$. For transitions from mode 0 to mode 2 the absolute value of the coefficient $c_{2}(t)$ must grow linearly in $t$ within perturbation theory. The expression (37) and therefore the propagator 34 feature terms growing linearly in $t$ if and only if at least one of the exponents on the right-hand side of (37) is zero. Taking into account $\left|\Delta \mathcal{E}_{n, n^{\prime}}^{++}\right|<\left|n-n^{\prime}\right| \omega$, this leads us to the unique resonance condition $\Delta \mathcal{E}_{0,2}^{++}=0$ which is equivalent to the classical energy-momentum conservation conditions (1) 
and (2) with $\boldsymbol{p}_{\text {in }}=\boldsymbol{p}$ and $n_{r}=1$ and $n_{l}=-1$. The second integral in 34 leads to the same resonance condition. Thus, on resonance the propagator $U_{\mathrm{nd} ; 2,0}^{++}(t, 0)$ reads in leading order in $t$

$$
U_{\mathrm{nd} ; 2,0}^{++}(t, 0)=-\frac{\mathrm{i} q^{2} t}{16} \mathrm{e}^{-\mathrm{i} \varepsilon_{2} t}\left(l^{+} L_{2,1}^{++} L_{1,0}^{++}+l^{-} L_{2,1}^{+-} L_{1,0}^{-+}\right)
$$

with the coefficients

$$
\begin{aligned}
& l^{+}=\frac{1}{\Delta \mathcal{E}_{0,1}^{++}-\omega}+\frac{1}{\Delta \mathcal{E}_{0,1}^{++}+\omega}, \\
& l^{-}=\frac{1}{\Delta \mathcal{E}_{0,1}^{+-}-\omega}+\frac{1}{\Delta \mathcal{E}_{0,1}^{+-}+\omega} .
\end{aligned}
$$

\subsection{The relativistic Rabi frequency}

Employing the explicit form of the matrices $L_{n, n^{\prime}}^{a b}$ in Eqs. (A3) and (A4) and the propagator (38) we calculate the diffraction probability $\left|c_{2}^{+\uparrow}(t)\right|^{2}+\left|c_{2}^{+\downarrow}(t)\right|^{2}$ which equals with $c_{n}^{+}(t)=$ $\left(c_{n}^{+\uparrow}(t), c_{n}^{+\downarrow}(t)\right)^{\top}$

$$
\begin{aligned}
\left|c_{2}^{+\uparrow}(t)\right|^{2}+\left|c_{2}^{+\downarrow}(t)\right|^{2}=c_{2}^{+}(t)^{\dagger} c_{2}^{+}(t) & \\
& =c_{2}^{+}(0)^{\dagger} U_{\mathrm{nd} ; 2,0}^{++}(t, 0)^{\dagger} U_{\mathrm{nd} ; 2,0}^{++}(t, 0) c_{2}^{+}(0) .
\end{aligned}
$$

Expanding $U_{\mathrm{nd} ; 2,0}^{++}(t, 0)$ from Eq. 38 in the momenta $k, p_{B}$, and $p_{E}$ yields

$$
\begin{aligned}
U_{\mathrm{nd} ; 2,0}^{++}(t, 0) & =-\frac{\mathrm{i} q^{2} t}{16} \frac{\hat{A}_{\max }^{2}}{m c^{2}} \frac{\mathrm{e}^{-\mathrm{i} \varepsilon_{2} t}}{m^{2} c^{2}}\left[\left(m^{2} c^{2}-\frac{p_{B}^{2}}{2}-\frac{5 p_{E}^{2}}{2}\right) \mathbb{1}\right. \\
& \left.+\frac{\mathrm{i} k p_{B}}{2} \sigma_{E}+\frac{\mathrm{i} 3 k p_{E}}{2} \sigma_{B}\right]+O\left(k^{3}, p_{E}^{3}, p_{B}^{3}\right) .
\end{aligned}
$$

Taking advantage of the normalization of the initial state, i. e., $\left|c_{0}^{+\uparrow}(0)\right|^{2}+\left|c_{0}^{+\downarrow}(0)\right|^{2}=1$, we finally get with (40) and (41)

$$
\left|c_{2}^{+\uparrow}(t)\right|^{2}+\left|c_{2}^{+\downarrow}(t)\right|^{2}=\frac{t^{2}}{4}\left(\frac{q^{2} \hat{E}^{2}}{8 k^{2} m c^{2}}\right)^{2}
$$

in leading order in $k, p_{B}$, and $p_{E}$. For times of the order of $8 k^{2} m c^{2} /\left(q^{2} \hat{E}^{2}\right)$ or larger we leave the domain where our timedependent perturbation theory is valid. Thus, the result (42) is correct for times much shorter than $8 k^{2} m c^{2} /\left(q^{2} \hat{E}^{2}\right)$ only. Making the ansatz

$$
\begin{aligned}
\left|c_{0}^{+\uparrow}(t)\right|^{2} & =\cos ^{2}\left(\frac{\Omega_{R} t}{2}\right), \\
\left|c_{2}^{+\uparrow}(t)\right|^{2}+\left|c_{2}^{+\downarrow}(t)\right|^{2} & =\sin ^{2}\left(\frac{\Omega_{R} t}{2}\right)
\end{aligned}
$$

for the long-time behavior, expanding this ansatz for short times, and comparing it to 42 gives the known Rabi frequency of the two-photon Kapitza Dirac effect [2, 40]

$$
\Omega_{R, 2}=\frac{q^{2} \hat{E}^{2}}{8 k^{2} m c^{2}} .
$$

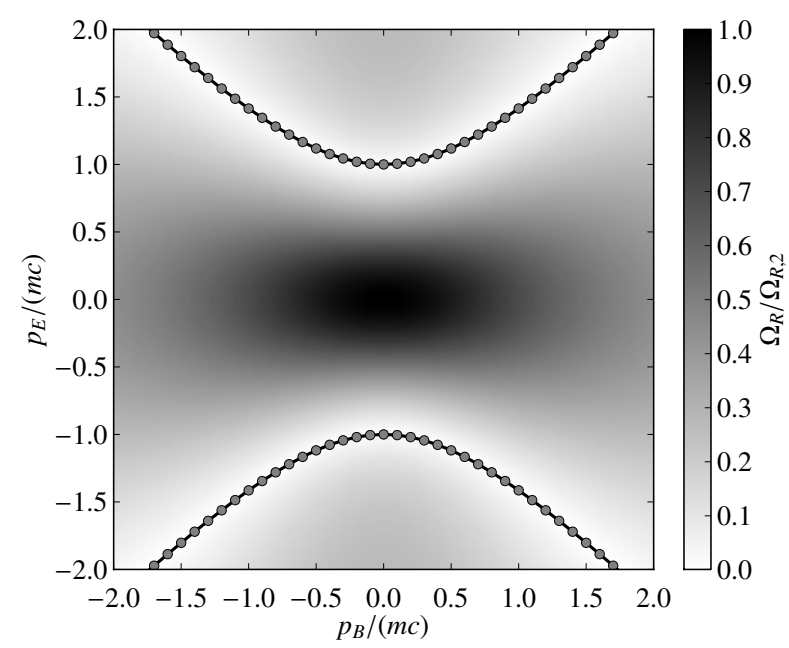

Figure 4: The relativistic Rabi frequency $\Omega_{R}$ of the two-photon Kapitza-Dirac effect normalized to the nonrelativistic Rabi frequency $\Omega_{R, 2}$ in Eq. 44. The applied parameters correspond to a photon energy of $12.5 \mathrm{keV}$. The gray filled circles indicate momenta for which the condition (49) is fulfilled and full spin flips can be observed. These momenta can be approximated by Eq. 50 (solid black line).

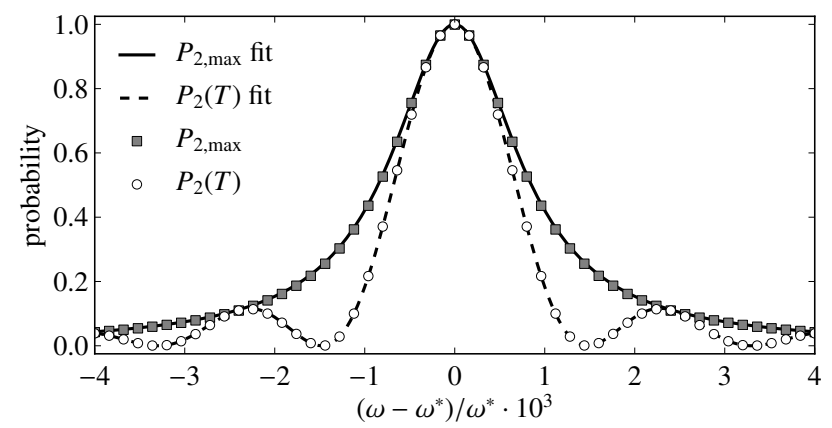

Figure 5: The off-resonant scattering probability $P_{2}(T)=\left|c_{2}^{+\uparrow}(T)\right|^{2}+$ $\left|c_{2}^{+\downarrow}(T)\right|^{2}$ at time $T=\pi / \Omega_{R}$ (half a Rabi cycle on resonance) and maximal scattering probability maximized over the total interaction time $P_{2, \max }=\max _{T^{\prime}} P_{2}\left(T^{\prime}\right)$ as functions of the relative detuning $\left(\omega-\omega^{*}\right) / \omega^{*}$. Dots and squares represent numerical data while lines are obtained via a fit to 45 and 46 yielding $b=29.2$ for the fitting parameter.

The expression (44) for the Rabi frequency which is based on the expansion (41) is valid only in the nonrelativistic domain. The Rabi frequency for relativistic momenta $k, p_{B}$, and $p_{E}$ can be calculated, however, with the help of computer algebra and numerical methods in a similar fashion as 44 by evaluating (40) and employing the fully relativistic propagator (38). The relativistic Rabi frequency is shown Fig. 4. Generally the relativistic Rabi frequency is lower than the nonrelativistic result (44). For the parameters as applied in the setup of Fig. 3 the theoretical relativistic Rabi frequency is $\Omega_{R}=7.241 \times$ $10^{15} \mathrm{~Hz}$, while numerically we obtain $\Omega_{R}=7.237 \times 10^{15} \mathrm{~Hz}$, which is in a fair agreement with the theoretical prediction.

Let $k^{*}$ denote an electron momentum and a photon mo- 
mentum that fulfill the Bragg condition (6). When the Bragg condition (6) is not exactly fulfilled by shifting the photon momentum $k$ from $k^{*}$, electrons scatter off-resonantly, leading to a modification of the Rabi frequency and a reduction of the maximal scattering probability. In the resonant two-photon Kapitza-Dirac effect only modes 0 and 2 are populated. Thus, the quantum system behaves similarly to an effective two-level system. This leads us to the off-resonant generalization

$$
\left|c_{2}^{+\uparrow}(T)\right|^{2}+\left|c_{2}^{+\downarrow}(T)\right|^{2}=\frac{\Omega_{R}^{2}}{\Omega^{2}} \sin ^{2}\left(\frac{\Omega T}{2}\right)
$$

of (25b) with the off-resonant Rabi frequency [53]

$$
\Omega=\sqrt{\Omega_{R}^{2}+\frac{\left(\omega-\omega^{*}\right)^{2}}{b^{2}}},
$$

$\omega=k c, \omega^{*}=k^{*} c$, and the parameter $b$ that accounts for the fact that the two-photon Kapitza-Dirac effect is not a pure two-level system. Numerical simulations indicate that the parameter $b$ varies with the laser frequency and the electron momentum and has the value $b=29.2$ for $p_{B}=0$ and $p_{E}=$ $1.00012 m c$ and $e \hat{A}_{\max }=2.2 \times 10^{-2} m c^{2}$. The off-resonant scattering probability $P_{2}(T)=\left|c_{2}^{+\uparrow}(T)\right|^{2}+\left|c_{2}^{+\downarrow}(T)\right|^{2}$ at time $T=$ $\pi / \Omega_{R}$ (half a Rabi cycle on resonance) and maximal scattering probability maximized over the total interaction time $P_{2, \max }=$ $\max _{T^{\prime}} P_{2}\left(T^{\prime}\right)$ obtained via numerical simulations are shown in Fig. 5 as functions of the relative detuning $\left(\omega-\omega^{*}\right) / \omega^{*}$. These numerical results can be fitted to (45) and (46), respectively, leading to the value $b=29.2$.

\subsection{Spin flips}

The propagator 41) features spin-preserving terms (proportional to $\mathbb{1}$ ) and spin-flipping terms (proportional to $\sigma_{E}$ and $\left.\sigma_{B}\right)$. If the condition

$$
m^{2} c^{2}-\frac{1}{2} p_{B}^{2}-\frac{5}{2} p_{E}^{2}=0
$$

is met, the propagator 41) predicts that spin-preserving transitions are totally suppressed; thus, a spin-flipping dynamics may become observable in the two-photon Kapitza-Dirac effect. However, this condition corresponds to an ellipse with major axis $p_{B}=\sqrt{2} m c$ and minor axis $p_{E}=\sqrt{2 / 5} m c$ and, therefore, we are beyond the validity of the nonrelativistic propagator 41). Although Eq. 47) is not a valid condition for spin-flipping transitions its derivation gives us a hint as to how to calculate the proper condition. In analogy to (41) one can expand the relativistic propagator (38) as a superposition of the matrices $\mathbb{1}, \sigma_{k}, \sigma_{B}$, and $\sigma_{E}$. With the help of the Frobenius inner product one can write

$$
\begin{aligned}
& U_{\mathrm{nd} ; 2,0}^{++}(t, 0)=\operatorname{tr}\left(\mathbb{1}^{\dagger} U_{\mathrm{nd} ; 2,0}^{++}(t, 0)\right) \frac{1}{2} \mathbb{1}+\operatorname{tr}\left(\sigma_{k}^{\dagger} U_{\mathrm{nd} ; 2,0}^{++}(t, 0)\right) \frac{1}{2} \sigma_{k} \\
& +\operatorname{tr}\left(\sigma_{B}^{\dagger} U_{\mathrm{nd} ; 2,0}^{++}(t, 0)\right) \frac{1}{2} \sigma_{B}+\operatorname{tr}\left(\sigma_{E}^{\dagger} U_{\mathrm{nd} ; 2,0}^{++}(t, 0)\right) \frac{1}{2} \sigma_{E}
\end{aligned}
$$

Thus, spin-preserving transitions are expected to be suppressed for

$$
\operatorname{tr}\left(\mathbb{1}^{\dagger} U_{\mathrm{nd} ; 2,0}^{++}(t, 0)\right)=0
$$

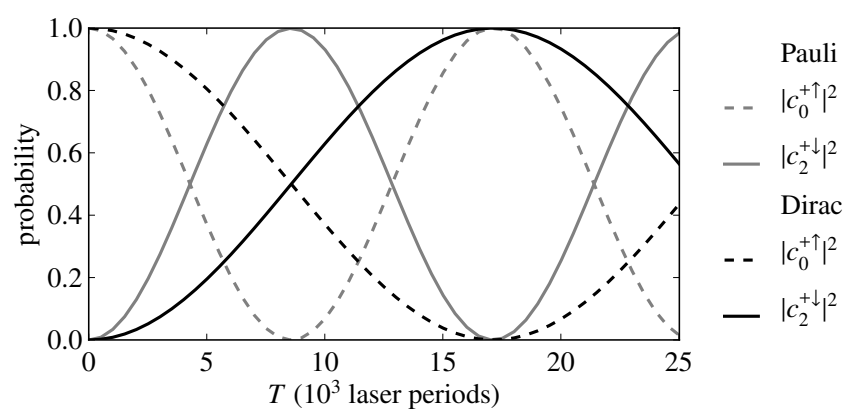

Figure 6: The two-photon Kapitza-Dirac effect with electron momenta chosen such that the spin-flip condition is fulfilled simulated by employing the Dirac equation (21) (black solid and dashed lines) and the Pauli equation (16) (gray solid and dashed lines). The electron interacts with a standing laser field of peak intensity $3 \times 10^{22} \mathrm{~W} / \mathrm{cm}^{2}$ (corresponding to $e \hat{A}_{\max }=2.2 \times 10^{-2} \mathrm{mc}^{2}$ ) for each beam and photon energy of $12.5 \mathrm{keV}$. The electron is diffracted to mode 2 , as in Fig. 3 However, because the initial electron momentum $\left(p_{B}=0\right.$, $p_{E}=1.00012 m c$ ) fulfills the spin-flip condition the electron spin changes its spin orientation during the diffraction process.

Again this condition can be evaluated with the help of computer algebra and numerical methods. The result is indicated in Fig. 4 by gray filled circles. These points lie approximately on the hyperbola (black solid line in Fig. 4)

$$
m^{2} c^{2}+p_{B}^{2}-p_{E}^{2}=0
$$

which can be seen as the fully relativistic version of the condition (47).

Employing time-dependent perturbation theory for the Pauli equation one can show that $m^{2} c^{2}-p_{E}^{2}=0$ is the corresponding condition for spin flips in the two-photon Kapitza-Dirac effect under the dynamics of the Pauli equation. Thus, the parameter setting $p_{B}=0$ and $p_{E}=m c$ fulfills the conditions for spinflipping electron scattering under the dynamics of the Dirac equation as well as under the Pauli equation, as demonstrated in Fig. 6, In contrast to the setting in Fig. 3, the Pauli equation and the Dirac equation yield different Rabi frequencies due to the relativistic electron momenta, for which the Pauli equation is actually not applicable. The hyperbola 50 lies approximately in a local minimum of the Rabi frequency (see Fig. 4). The Rabi frequency along the gray filled dots in Fig. 4 is shown in Fig. 7. For photon momenta $k$ that are small compared to $m c$ the Rabi frequency drops down by two orders of magnitude compared to $\Omega_{R, 2}$, which is a consequence of the relative strengths of the spin-preserving and the spin-flipping terms in 41).

Because the basis functions 18 are eigenfunctions of the Foldy-Wouthuysen spin operator in the laser's polarization direction the coefficients $c_{2}^{+\uparrow}(t)$ and $c_{2}^{+\downarrow}(t)$ give immediately the spin of the diffracted beam if one chooses the electric field direction as quantization axis. Perturbation theory allows us also to calculate the orientation of the spin in the diffracted part of the electron beam. More precisely, we ask the question: What is the expectation value of the spin $s_{\text {out }}$ in the scattered beam if the beam of incident electrons has spin $s_{\text {in }}$ ? 


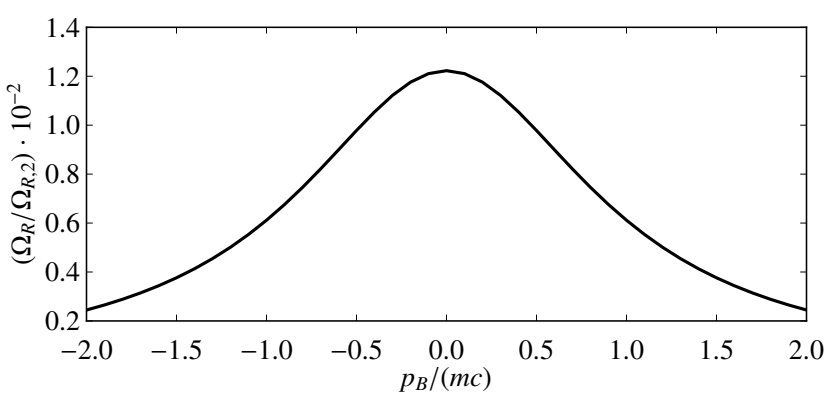

Figure 7: Relativistic Rabi frequency of the two-photon KapitzaDirac effect at momenta for which full spin flips are possible (that means along the gray filled circles in Fig. 4).

The electron spin is determined by applying the FoldyWouthuysen spin operator to the quantum state. In our momentum-space representation the expectation value of the Foldy-Wouthuysen spin operator is

$$
s=\frac{1}{2} \sum_{n} c_{n}(t)^{\dagger} \Sigma c_{n}(t)
$$

with $\Sigma=\left(\Sigma_{1}, \Sigma_{2}, \Sigma_{3}\right)^{\top}$ and

$$
\Sigma_{i}=\left(\begin{array}{cc}
\sigma_{i} & 0 \\
0 & \sigma_{i}
\end{array}\right) .
$$

The incident electron beam has well-defined momentum but may have arbitrary spin orientation; thus it can be expressed as a superposition of two positive-energy states with the same momentum. Introducing the Bloch angles $\theta$ and $\phi$ we may write the initial state as

$$
c_{0}(0)=\left(\begin{array}{c}
c_{0}^{+\uparrow}(0) \\
c_{0}^{+\downarrow}(0) \\
c_{0}^{-\uparrow}(0) \\
c_{0}^{-\downarrow}(0)
\end{array}\right)=\left(\begin{array}{c}
\cos (\theta / 2) \\
\sin (\theta / 2) \mathrm{e}^{\mathrm{i} \phi} \\
0 \\
0
\end{array}\right)
$$

and $c_{n}(0)=(0,0,0,0)^{\top}$ for all $n \neq 0$. The spin expectation value of the initial quantum state is

$$
\boldsymbol{s}_{\text {in }}=\frac{1}{2}\left(\begin{array}{c}
\sin (\theta) \cos (\phi) \\
\sin (\theta) \sin (\phi) \\
\cos (\theta)
\end{array}\right) .
$$

The spin expectation value of the diffracted part is

$$
\boldsymbol{s}_{\mathrm{out}}=\frac{1}{2} \frac{c_{2}(t)^{\dagger} \boldsymbol{\Sigma} c_{2}(t)}{c_{2}(t)^{\dagger} c_{2}(t)} .
$$

Employing the time evolution operator 38 we find

$$
\boldsymbol{s}_{\mathrm{out}}=\frac{1}{2} \frac{c_{0}^{+\dagger}(0) U_{\mathrm{nd} ; 2,0}^{++\dagger} \sigma U_{\mathrm{nd} ; 2,0}^{++} c_{0}^{+}(0)}{c_{0}^{+\dagger}(0) U_{\mathrm{nd} ; 2,0}^{++\dagger} U_{\mathrm{nd} ; 2,0}^{++} c_{0}^{+}(0)} .
$$

The time evolution operator (38) is up to a multiplicative factor a unitary $2 \times 2$ matrix and, therefore, may be witten as

$$
U_{\mathrm{nd} ; 2,0}^{++}=\sqrt{P} \mathrm{e}^{\mathrm{i} \phi}\left[\cos \left(\frac{\gamma}{2}\right) \mathbb{1}-\mathrm{i} \sin \left(\frac{\gamma}{2}\right) \boldsymbol{n}_{\mathrm{r}} \cdot \boldsymbol{\sigma}\right]
$$

with the real-valued parameters $\gamma, \boldsymbol{n}_{\mathrm{r}}$, and $P$ which can be determined via equating coefficients in (57) and (38). The expression in the square brackets is the SU(2) representation of a rotation around the rotation axis given by the unit vector $\boldsymbol{n}_{\mathrm{r}}$ and the rotation angle $\gamma$. Thus, the electron's spin orientation after diffraction $\boldsymbol{s}_{\text {out }}$ results from a rotation of $s_{\text {in }}$ around the axis $\boldsymbol{n}_{\mathrm{r}}$ by the angle $\gamma$.

For nonrelativistic electron momenta the parameters of this rotation can be uniquely identified by equating coefficients in (57) and (41). We find the rotation axis

$$
\boldsymbol{n}_{\mathrm{r}}=-\frac{1}{\sqrt{9 p_{E}^{2}+p_{B}^{2}}}\left(\begin{array}{c}
0 \\
3 p_{E} \\
p_{B}
\end{array}\right)
$$

and the rotation angle

$$
\gamma=2 \arctan \left(\frac{k \sqrt{9 p_{E}^{2}+p_{B}^{2}}}{2 m^{2} c^{2}-p_{B}^{2}-5 p_{E}^{2}}\right) .
$$

Thus, for nonrelativistic momenta $k, p_{E}$, and $p_{B}$ only very small spin rotations may be observed in the two-photon Kapitza-Dirac effect.

\section{Three-photon Kapitza-Dirac effect}

\subsection{Numerical results}

According to the semi-classical considerations in Sec. 2, two photons are absorbed from the laser field and one photon is emitted into the laser field in the three-photon Kapitza-Dirac

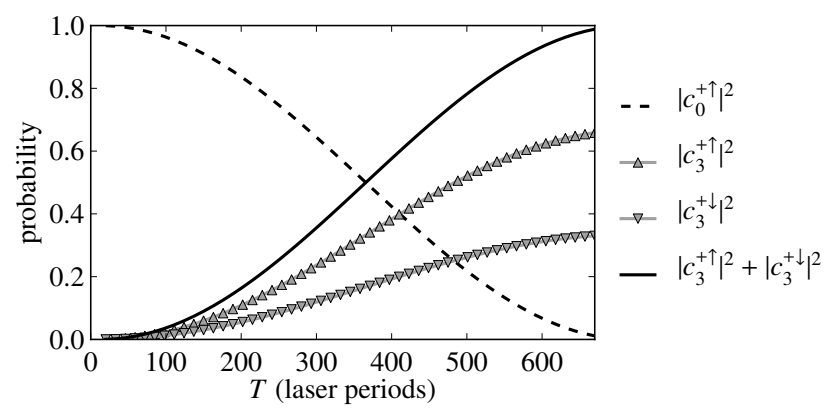

Figure 8: The three-photon Kapitza-Dirac effect simulated by employing the Dirac equation 21] for one half of a Rabi cycle. The electron with initial momentum $176 \mathrm{keV}=0.347 \mathrm{mc}$ in the laser propagation direction (dashed line) is diffracted to the final momentum $177 \mathrm{keV}=0.365 \mathrm{mc}$ in the laser propagation direction (solid line) by its interaction with a standing light wave with peak intensity $2 \times 10^{23} \mathrm{~W} / \mathrm{cm}^{2}$ (corresponding to $e \hat{A}_{\max }=0.21 m c^{2}$ ) for each beam and photon momentum $3.1 \mathrm{keV} / \mathrm{c}=6.1 \times 10^{-3} \mathrm{mc}$. The electron momentum in the laser polarization direction is $1.2 \mathrm{keV}=2.4 \times 10^{-3} \mathrm{mc}$. The probability of the diffracted electron thereby splits up into a spinflipped part (downward triangles) and a spin-preserving part (upward triangles). Data adopted from [39]. 


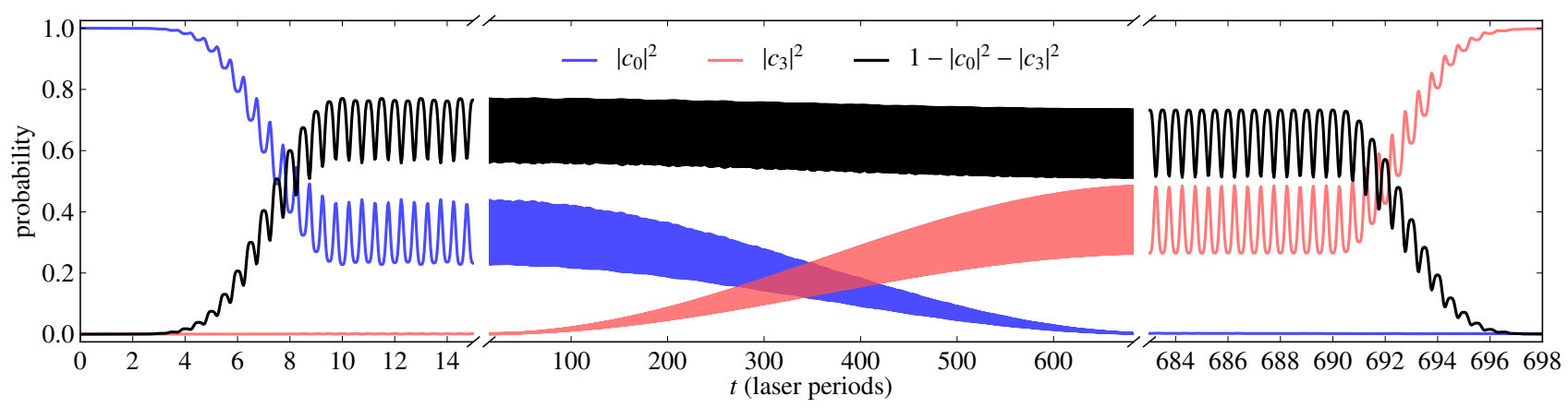

Figure 9: (Color online) The in-field quantum dynamics of the three-photon Kapitza-Dirac effect. The graph shows the occupation probability of the unscattered beam $\left|c_{0}\right|^{2}$ and of the diffracted beam $\left|c_{3}\right|^{2}$, and the remaining occupation probability $1-\left|c_{0}\right|^{2}-\left|c_{3}\right|^{2}$ of all other modes. The occupation probabilities oscillate in the laser field with twice the laser frequency. The laser field is shaped by a turn-on envelope of ten laser cycles (left part of the plot) followed by a period of 678 laser cycles with constant laser intensity (center part of the plot) and a turn-off envelope of ten laser cycles (right part of the plot); see (9). When the laser is at maximal intensity the probability of finding the electron at a certain momentum is distributed over the neighboring modes of the modes 0 and 3 . After the final turn-off, however, only the modes 0 and 3 are occupied. The relative occupation of modes 0 and 3 depends on the total interaction time $T$; see Fig. 8 . Here $T$ has been chosen such that $\left|c_{0}(T)\right|^{2} \approx 0$ and $\left|c_{3}(T)\right|^{2} \approx 1$.

effect, corresponding to $n_{r}=2$ and $n_{l}=-1$. This particular Kapitza-Dirac effect has been examined in our recent publication [39] where we demonstrated that explicit spin dynamics can be observed depending on the electron's and the laser's parameters. Here we will investigate the three-photon Kapitza-Dirac effect in more detail, analyzing, for example, the in-field dynamics, conditions for full spin flips, and the Rabi frequency at relativistic electron momenta. For the readers' convenience we show in Fig. 8 the time evolution of the occupation $\left|c_{n}^{\zeta}(t)\right|^{2}$ governed by the Dirac equation in momentum space 21) for the parameters that have also been considered in [39]. The parameters of the simulation are a photon momentum of $k=6.1 \times 10^{-3} \mathrm{mc}$, an electron momentum of $p_{E}=2.4 \times 10^{-3} \mathrm{mc}$ in the laser polarization direction, an electron momentum of $p_{k}=0.347 \mathrm{mc}$ in the laser propagation direction and a field amplitude of $e \hat{A}_{\max }=0.21 m c^{2}$. Starting from the initial condition (24) the quantum dynamics features Rabi oscillations in the form

$$
\begin{array}{r}
\left|c_{0}^{+\uparrow}(T)\right|^{2}=\cos ^{2}\left(\frac{\Omega_{R} T}{2}\right), \\
\left|c_{3}^{+\uparrow}(T)\right|^{2}+\left|c_{3}^{+\downarrow}(T)\right|^{2}=\sin ^{2}\left(\frac{\Omega_{R} T}{2}\right),
\end{array}
$$

similarly to the two-photon Kapitza-Dirac effect. However, a fraction of 0.33 of the diffracted electrons have flipped their spin with respect to the quantization axis in the laser polarization direction.

In contrast to the quantum state after the interaction with the laser (shown in Fig. 8), also the neighboring modes of the modes 0 and 3 are occupied during the in-field quantum dynamics; see Fig. 9. During the turn-on phase the quantum state populates the neighboring modes of mode 0 and in the following interaction with the laser at constant intensity mode 3 gets partly occupied. After turn-off, however, only the modes 0 and 3 have a significant occupation probability and all other occupation probabilities vanish.

\subsection{Perturbation theory}

In this section we derive a perturbative short-time solution of the three-photon Kapitza-Dirac effect, for obtaining analytic expressions for the Rabi frequency and the electron spin-flip probability, as in the two-photon Kapitza-Dirac effect. Analogously to the two-photon Kapitza-Dirac effect, we want to approximate the propagator $U_{3,0}(t, 0)$, which maps the initial quantum state $c_{0}(0)$ to the final quantum state by $c_{3}(t)$ by

$$
c_{3}(t)=U_{3,0}(t, 0) c_{0}(0) .
$$

Since the Dirac equation contains only couplings to the nextneighboring modes, the lowest-order perturbative solution is of third order and reads

$$
U_{\mathrm{rd}}(t, 0)=\frac{1}{\mathrm{i}^{3}} \int_{0}^{t} \mathrm{~d} t_{3} \int_{0}^{t_{3}} \mathrm{~d} t_{2} \int_{0}^{t_{2}} \mathrm{~d} t_{1} U_{0}\left(t, t_{3}\right) V\left(t_{3}\right) U_{0}\left(t_{3}, t_{2}\right) V\left(t_{2}\right) U_{0}\left(t_{2}, t_{1}\right) V\left(t_{1}\right) U_{0}\left(t_{1}, 0\right) .
$$

Utilizing the explicit form of the free propagator (29) and the interaction Hamiltonian (30) in momentum space yields the time-dependent perturbation theory propagator 


$$
\begin{aligned}
U_{\mathrm{rd} ; 3,0}(t, 0)=\frac{\mathrm{i} q^{3}}{8} L_{3,2}^{++} & L_{2,1}^{++} L_{1,0}^{++} \int_{0}^{t} \mathrm{~d} t_{3} \int_{0}^{t_{3}} \mathrm{~d} t_{2} \int_{0}^{t_{2}} \mathrm{~d} t_{1} \sin \left(\omega t_{3}\right) \sin \left(\omega t_{2}\right) \sin \left(\omega t_{1}\right) v^{++}\left(t, t_{3}, t_{2}, t_{1}\right) \\
& +\frac{\mathrm{i} q^{3}}{8} L_{3,2}^{+-} L_{2,1}^{-+} L_{1,0}^{++} \int_{0}^{t} \mathrm{~d} t_{3} \int_{0}^{t_{3}} \mathrm{~d} t_{2} \int_{0}^{t_{2}} \mathrm{~d} t_{1} \sin \left(\omega t_{3}\right) \sin \left(\omega t_{2}\right) \sin \left(\omega t_{1}\right) v^{-+}\left(t, t_{3}, t_{2}, t_{1}\right) \\
& +\frac{\mathrm{i} q^{3}}{8} L_{3,2}^{++} L_{2,1}^{+-} L_{1,0}^{-+} \int_{0}^{t} \mathrm{~d} t_{3} \int_{0}^{t_{3}} \mathrm{~d} t_{2} \int_{0}^{t_{2}} \mathrm{~d} t_{1} \sin \left(\omega t_{3}\right) \sin \left(\omega t_{2}\right) \sin \left(\omega t_{1}\right) v^{+-}\left(t, t_{3}, t_{2}, t_{1}\right) \\
& +\frac{\mathrm{i} q^{3}}{8} L_{3,2}^{+-} L_{2,1}^{--} L_{1,0}^{-+} \int_{0}^{t} \mathrm{~d} t_{3} \int_{0}^{t_{3}} \mathrm{~d} t_{2} \int_{0}^{t_{2}} \mathrm{~d} t_{1} \sin \left(\omega t_{3}\right) \sin \left(\omega t_{2}\right) \sin \left(\omega t_{1}\right) v^{--}\left(t, t_{3}, t_{2}, t_{1}\right),
\end{aligned}
$$

with the phases

$$
\begin{aligned}
& v^{a b}\left(t, t_{3}, t_{2}, t_{1}\right)= \\
& \quad \exp \left[-\mathrm{i}\left(\mathcal{E}_{3} t+\Delta \mathcal{E}_{2,3}^{a+} t_{3}+\Delta \mathcal{E}_{1,2}^{b a} t_{2}+\Delta \mathcal{E}_{0,1}^{+b} t_{1}\right)\right],
\end{aligned}
$$

where the upper indices $a$ and $b$ take the values + and -. Analogously to Sec. 4.2, the integrand of the time integral

$$
\int_{0}^{t} \mathrm{~d} t_{3} \int_{0}^{t_{3}} \mathrm{~d} t_{2} \int_{0}^{t_{2}} \mathrm{~d} t_{1} \sin \left(\omega t_{3}\right) \sin \left(\omega t_{2}\right) \sin \left(\omega t_{1}\right) v^{a b}\left(t, t_{3}, t_{2}, t_{1}\right)
$$

also contains oscillating terms which become constant if the resonance condition of the three-photon Kapitza-Dirac effect $\mathcal{E}_{3}-\mathcal{E}_{0}=\omega$ is met. Accounting only for the terms which grow linearly in time yields the propagator

$$
\begin{aligned}
& U_{\mathrm{rd} ; 3,0}^{++}(t, 0)= \\
& \quad\left(-\frac{q^{3} t}{64}\right) \mathrm{e}^{-\mathrm{i} \mathcal{E}_{3} t}\left(l^{++} L_{3,2}^{++} L_{2,1}^{++} L_{1,0}^{++}+l^{-+} L_{3,2}^{+-} L_{2,1}^{-+} L_{1,0}^{++}\right. \\
& \left.+l^{+-} L_{3,2}^{++} L_{2,1}^{+-} L_{1,0}^{-+}+l^{--} L_{3,2}^{+-} L_{2,1}^{--} L_{1,0}^{-+}\right),
\end{aligned}
$$

with the coefficients

$$
\begin{aligned}
l^{a b}=\frac{1}{\Delta E_{0,1}^{+b}-\omega} \frac{1}{\Delta E_{0,2}^{+a}}+ & \frac{1}{\Delta E_{0,1}^{+b}+\omega} \frac{1}{\Delta E_{0,2}^{+a}} \\
& +\frac{1}{\Delta E_{0,1}^{+b}+\omega} \frac{1}{\Delta E_{0,2}^{+a}+2 \omega} .
\end{aligned}
$$

\subsection{The relativistic Rabi frequency}

An expansion of the propagator $U_{\mathrm{rd} ; 3,0}^{++}(t, 0)$ in Eq. 66 with respect to the momenta $k, p_{B}$ and $p_{E}$ yields

$$
\begin{aligned}
& U_{\mathrm{rd} ; 3,0}^{++}(t, 0)= \\
& \begin{aligned}
\frac{q^{3} t}{48} \frac{\hat{A}_{\max }^{3}}{m^{2} c^{4}} \frac{\mathrm{e}^{-\mathrm{i} \varepsilon_{3} t}}{m c}\left(\frac{5 p_{E}}{\sqrt{2}} \mathbb{1}-\mathrm{i} k \sigma_{B}-\right. & \left.\mathrm{i} \frac{3-2 \sqrt{2}}{m c} k p_{B} \sigma_{k}\right) \\
+ & O\left(k^{3}, p_{E}^{3}, p_{B}^{3}\right) .
\end{aligned}
\end{aligned}
$$

Evaluating $\left|c_{3}^{+\uparrow}(t)\right|^{2}+\left|c_{3}^{+\downarrow}(t)\right|^{2}$ in analogy to 40 with 61) and (68) yields the short-time diffraction probability

$$
\begin{aligned}
& \left|c_{3}^{+\uparrow}(t)\right|^{2}+\left|c_{3}^{+\downarrow}(t)\right|^{2}= \\
& \frac{t^{2}}{4}\left(\frac{q^{3} \hat{E}^{3}}{24 m^{3} c^{5} k^{3}}\right)^{2}\left[\frac{25}{2} p_{E}^{2}+k^{2}+(3-2 \sqrt{2}) k^{2} \frac{p_{B}^{2}}{m^{2} c^{2}}\right] .
\end{aligned}
$$

By comparing this probability with the analogous short-time expansion of the ansatz 43, , one finds the Rabi frequency

$$
\Omega_{R}=\Omega_{R, 3} \sqrt{\frac{25}{2} \frac{p_{E}^{2}}{k^{2}}+1+(3-2 \sqrt{2}) \frac{p_{B}^{2}}{m^{2} c^{2}}},
$$

with the Rabi frequency

$$
\Omega_{R, 3}=\frac{q^{3} \hat{E}^{3}}{24 m^{3} c^{5} k^{2}}
$$

The expression (70) holds only for small $k, p_{E}$, and $p_{B}$. However, one may evaluate $\left|c_{3}^{+\uparrow}(t)\right|^{2}+\left|c_{3}^{+\downarrow}(t)\right|^{2}$ with the exact relativistic propagator (66), which is shown in Fig. 10. The common property of the Rabi frequencies $\Omega_{R, 2}$ and $\Omega_{R, 3}$ is that both hold at the origin $p_{E}=p_{B}=0$ of the $p_{E}-p_{B}$ plane. The difference between the two is that the two-photon KapitzaDirac effect shows no spin flip for $p_{E}=p_{B}=0$ and the Rabi frequency is maximal for these momenta. In the three-photon Kapitza-Dirac effect $p_{E}=p_{B}=0$ implies a full spin-flip position (see the next section) and the Rabi frequency has a saddle point. For the parameters which are used in Fig. 8 this Rabi frequency evaluates to $\Omega_{R}=3.43 \times 10^{15} \mathrm{~Hz}$ whereas the Rabi frequency from the simulation $\Omega_{R}=3.34 \times 10^{15} \mathrm{~Hz}$ agrees well with the analytical result.

In analogy to the two-photon Kapitza-Dirac effect, the offresonant diffraction probability (45) with the off-resonant Rabi frequency (46) also applies to the three-photon Kapitza-Dirac effect. For the parameters applied in Fig. 8 we find numerically $b=45.7$. We remark that in the case of the threephoton Kapitza-Dirac effect a systematic shift of the resonance peak appears in the numerical simulation, as compared to the peak position which we obtain from the classical resonance condition (6). For the parameters $p_{k}=0.3470 \mathrm{mc}$ and $p_{E}=2.4 \times 10^{-3} m c$ of Fig. 8 for example, one finds the resonance peak at the photon momentum $k=6.1 \times 10^{-3} \mathrm{mc}$ in the numerical simulation, whereas the resonance condition (6) predicts the photon momentum $k=4.4 \times 10^{-3} \mathrm{mc}$. This shift scales with the laser intensity, as the quantum dynamics leaves the perturbative regime with increasing field amplitude. Therefore, the resonance peak position of the numerical solution and the classical condition (6) converge to the same value in the limit of small laser intensities. 


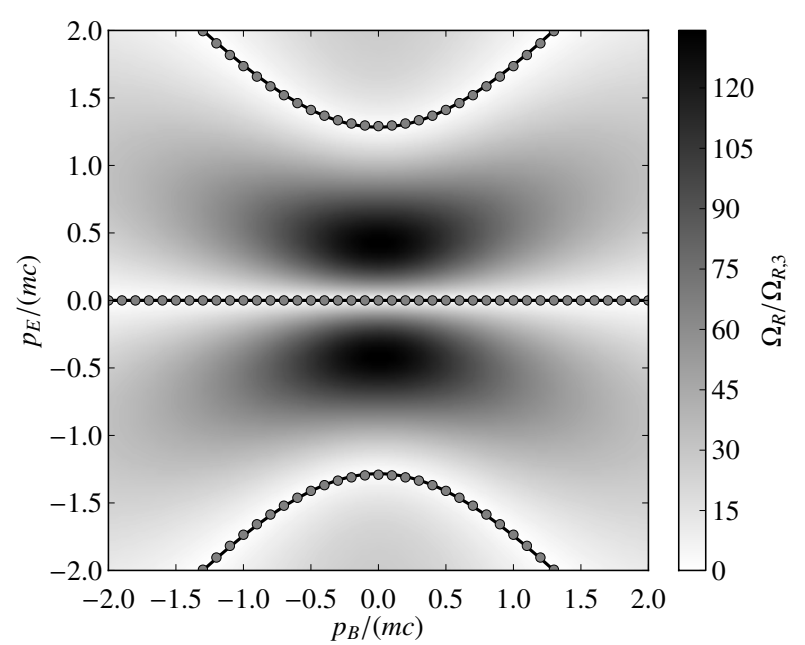

Figure 10: The relativistic Rabi frequency of the three-photon Kapitza-Dirac effect normalized to the full spin-flip Rabi frequency $\Omega_{R, 3}$ in Eq. (71). The parameters in this figure are a laser intensity of $2 \times 10^{23} \mathrm{~W} / \mathrm{cm}^{2}$ and a photon energy of $3.1 \mathrm{keV}$. The gray filled circles mark positions in which condition 72 is fulfilled, which are approximated by the Eqs. 73 .

\subsection{Spin flips}

As in Sec. 4.4, we identify the parameter space for spin-flips in the three-photon Kapitza-Dirac effect by the condition

$$
\operatorname{tr}\left(\mathbb{1}^{\dagger} U_{\mathrm{rd} ; 3,0}^{++}(t, 0)\right)=0 .
$$

The numerical evaluation of this condition yields the gray filled circles in Fig. 10 which form a line and a hyperbola. The line and the hyperbola can be approximated by the equations

$$
\begin{aligned}
p_{E} & =0, \\
m^{2} c^{2}+0.847 p_{B}^{2}-0.608 p_{E}^{2} & =0 .
\end{aligned}
$$

The line 73 a can also be derived from the expansion 68 , because the spin-flip condition runs through the point $p_{E}=$ $p_{B}=0$, and the Taylor expansion is exact in the vicinity of this point. We also plot the Rabi frequency at the regions of a full spin flip in Fig. 11, as in Sec. 4.4, the Rabi frequency of the spin-flip regions is about two orders of magnitude lower than the Rabi frequency of the spin-preserving regions.

In analogy to the two-photon Kapitza-Dirac effect the electron spin in the diffracted part of the laser beam can be expressed as

$$
\boldsymbol{s}_{\mathrm{out}}=\frac{1}{2} \frac{c_{0}^{+\dagger}(0) U_{\mathrm{rd} ; 3,0}^{++\dagger} \sigma U_{\mathrm{rd} ; 3,0}^{++} c_{0}^{+}(0)}{c_{0}^{+\dagger}(0) U_{\mathrm{rd} ; 3,0}^{++\dagger} U_{\mathrm{rd} ; 3,0}^{++} c_{0}^{+}(0)} .
$$

by utilizing the time evolution operator 66. For nonrelativistic electron momenta $p_{B}$ and $p_{E}$ the parameters of the rotation of the spin can be uniquely identified by equating coefficients in (57) and (68). In contrast to the two-photon Kapitza-Dirac effect we find significant spin rotations for the electron momenta

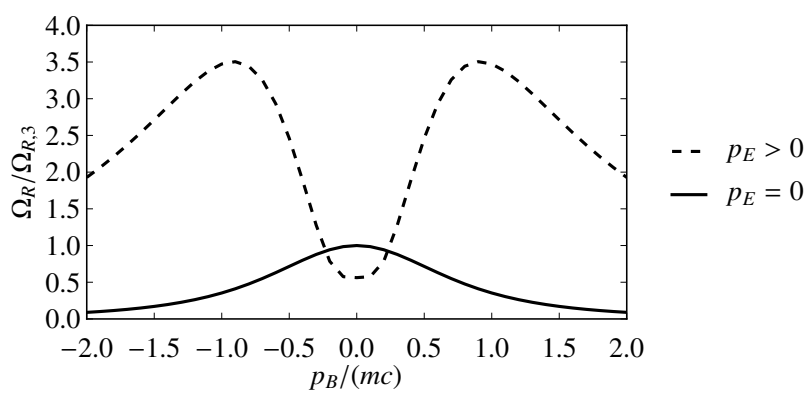

Figure 11: Relativistic Rabi frequency of the three-photon KapitzaDirac effect at momenta for which full spin-flips are possible (that means along the gray filled circles in Fig. 10,

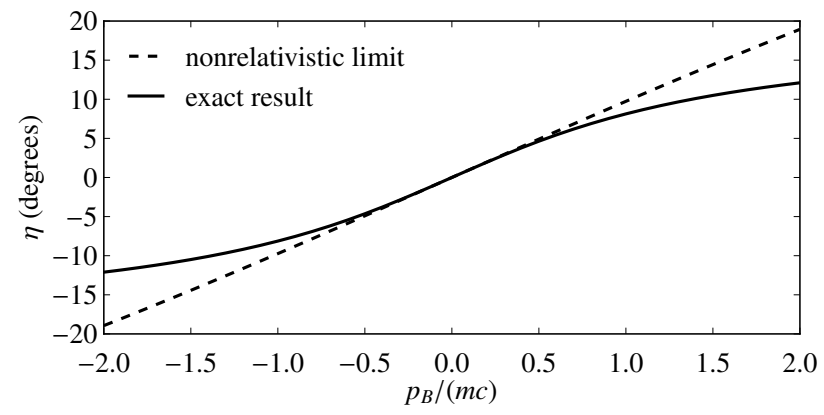

Figure 12: Tilt of the electron spin rotation axis $\boldsymbol{n}_{\mathrm{r}}$. The dashed line shows the analytical result 77 of the expanded propagator 68. The black line is obtained numerically from the exact propagator 66. The tilt of the rotation angle takes place in the plane spanned by $k$ and $\hat{B}$, because the $E$ component of the vector $[75$ and the $E$ component of the vector from the exact computation are zero.

$p_{E}$ and $p_{B}$. For the case of the three-photon Kapitza-Dirac effect, the rotation axis is given by

$$
\boldsymbol{n}_{\mathrm{r}}=\frac{1}{\sqrt{(3-2 \sqrt{2})^{2} p_{B}^{2}+m^{2} c^{2}}}\left(\begin{array}{c}
(3-2 \sqrt{2}) p_{B} \\
m c \\
0
\end{array}\right)
$$

and the rotation angle reads

$$
\gamma=2 \arctan \left[\frac{\sqrt{2}}{5} \frac{k}{p_{E}} \sqrt{1+(3-2 \sqrt{2})^{2} \frac{p_{B}^{2}}{m^{2} c^{2}}}\right] .
$$

Equation (75) means that the electron spin is rotated around the axis of the magnetic field, if the electron momentum $p_{B}$ in the magnetic field direction is zero. A nonvanishing $p_{B}$, however, tilts the rotation axis of the spin rotation into the direction of the laser propagation direction by the angle

$$
\eta \equiv \arctan \left(\frac{n_{\mathrm{r}, k}}{n_{\mathrm{r}, B}}\right)=\arctan \left[(3-2 \sqrt{2}) \frac{p_{B}}{m c}\right]
$$

This tilt angle $\eta$ is plotted together with the exact value, obtained from the propagator [66, in Fig. 12 


\section{Conclusions and Outlook}

We discussed the general relativistic and nonrelativistic Bragg conditions for a Kapitza-Dirac effect with $n$ interacting photons that follow from energy-momentum conservation. We investigated the two-photon and three-photon Kapitza-Dirac effects by accounting for the Bragg conditions and simulating the Pauli equation and the Dirac equation, which were transformed into a momentum-space representation. In both scenarios spin effects appear at relativistic momenta of the electron. We also presented an analytic solution of the Kapitza-Dirac effect by computing the short-time evolution with time-dependent perturbation theory for the two-photon and the three-photon Kapitza-Dirac effects. By the help of our analytical and numerical methods, we were able to demonstrate full spin flips within the two-photon Kapitza-Dirac effect. Furthermore, we pointed out that the spin flip in the Kapitza-Dirac effect corresponds to a rotation of the electron spin, when the electron is diffracted. The diffraction probability, however, does not depend on the spin orientation of the incident electrons. Our numerical simulations indicate that the off-resonant Kapitza-Dirac effect can be described by a generalization of Rabi theory for two-level systems.

The Rabi frequency of the $n$-photon Kapitza-Dirac effect scales with the $n$th power of $e \hat{E} /\left(k m c^{2}\right)$, because the lowest order contribution in time-dependent perturbation theory is of $n$th order and contains a product of $n$ times the interaction Hamiltonian (30). Therefore, the Rabi frequency of the three-photon Kapitza-Dirac effect is suppressed by a factor of $e \hat{E} /\left(k m c^{2}\right)$ as compared to the Rabi frequency of the two-photon KapitzaDirac effect. Note that $e \hat{E} / k$ is always smaller than $m c^{2}$ in the Bragg regime. Thus, from this point of view, higher laser intensities are in principle required for higher photon processes.

The two-photon and three-photon Kapitza-Dirac effects are different, if the two are compared against the background of spontaneous emission. Since spontaneous emission is proportional to the square of the electric field, the radiation power spontaneously emitted by the electron scales like the Rabi frequency of the two-photon Kapitza-Dirac effect with the square of $e \hat{E} /\left(k m c^{2}\right)$. This implies that the spontaneously emitted energy which is emitted in one Rabi cycle is independent of the laser intensity for the two-photon Kapitza-Dirac effect. The Rabi frequency of the three-photon Kapitza-Dirac effect, however, scales with the third power of $e \hat{E} /\left(k m c^{2}\right)$. Therefore, the three-photon Kapitza-Dirac effect and all higher-order KapitzaDirac effects may become only visible for very high intensities of the external laser field.

Even though the above considerations favor the two-photon Kapitza-Dirac effect for an experimental demonstration of spin effects, the two-photon Kapitza-Dirac effect has the drawback, that spin effects occur only for relativistic momenta of the injected electron in the laser polarization direction. This implies less favorable short interaction times of the electron with the laser, if the electron passes through a narrowly focused laser beam.

Our numerical solution of the quantum dynamics also shows that many modes are excited in the in-field dynamics, indicating that the results from perturbation theory might be appli- cable even for interaction parameters in which higher-order perturbative corrections should be of relevance. The good agreement of our numerical results with the perturbative approximation moreover suggests the applicability of our predictions in the parameter space of intense, optical laser beams. Therefore we conjecture that spin signatures in the KapitzaDirac effect might be realizable even for the interaction of moderately relativistic electrons with intense laser beams in the optical regime.

\section{Acknowledgments}

S. A. would like to thank Rainer Grobe for inspiring discussions and Matthias Dellweg for carefully reading the manuscript.

\section{A. Spin-interaction matrices}

To express the spin-interaction matrices $L_{n, n^{\prime}}^{a b}$ 32, we first introduce the coefficients

$$
\begin{aligned}
& d_{n}^{+}=\frac{1}{\sqrt{2}} \sqrt{\frac{\mathcal{E}_{n}+m c^{2}}{\mathcal{E}_{n}}} \\
& d_{n}^{-}=\frac{c}{\sqrt{2}} \sqrt{\frac{1}{\mathcal{E}_{n}\left(\mathcal{E}_{n}+m c^{2}\right)}},
\end{aligned}
$$

which allows us to define the coefficients

$$
\begin{aligned}
t_{n, n^{\prime}} & =d_{n}^{+} d_{n^{\prime}}^{+}+\boldsymbol{p}_{n} \cdot \boldsymbol{p}_{n^{\prime}} d_{n}^{-} d_{n^{\prime}}^{-}, \\
s_{n, n^{\prime}}^{l} & =p_{n}^{l} d_{n}^{-} d_{n^{\prime}}^{+}+p_{m}^{l} d_{n}^{+} d_{n^{\prime}}^{-}, \\
r_{n, n^{\prime}}^{l} & =p_{n}^{l} d_{n}^{-} d_{n^{\prime}}^{+}-p_{n^{\prime}}^{l} d_{n}^{+} d_{n^{\prime}}^{-}, \\
w_{n, n^{\prime}}^{l, q} & =p_{n}^{l} p_{n^{\prime}}^{q} d_{n}^{-} d_{n^{\prime}}^{-}+p_{n}^{q} p_{n^{\prime}}^{l} d_{n}^{-} d_{n^{\prime}}^{-}, \\
h_{n, n^{\prime}}^{l} & =\boldsymbol{e}_{l} \cdot\left(\boldsymbol{p}_{n} \times \boldsymbol{p}_{n^{\prime}}\right) d_{n}^{-} d_{n^{\prime}}^{-} .
\end{aligned}
$$

The upper indices denote the vector components of the coefficients, whereas the lower indices correspond to a mode number of the wave function (13) of the electron. One can show [54] that the matrices $L_{n, n^{\prime}}^{a b}$ in (32) are given by

$$
L_{n, n^{\prime}}^{++}=-L_{n, n^{\prime}}^{--}=\sum_{l} \hat{A}^{l}(t) s_{n, n^{\prime}}^{l} \mathbb{1}+\mathrm{i} \sum_{l q j} \epsilon_{j l q} r_{n, n^{\prime}}^{l} \hat{A}^{q}(t) \sigma^{j}
$$

and

$$
\begin{aligned}
& L_{n, n^{\prime}}^{+-}=L_{n, n^{\prime}}^{-+} \\
& \quad=\sum_{l} t_{n, n^{\prime}} \hat{A}^{l}(t) \sigma^{l}-\sum_{l, q} w_{n, n^{\prime}}^{l, q} \hat{A}^{q}(t) \sigma^{l}+\mathrm{i} \sum_{l} \hat{A}^{l}(t) h_{n, n^{\prime}}^{l} \mathbb{1} .
\end{aligned}
$$


[1] P. L. Kapitza and P. A. M. Dirac, Math. Proc. Cambridge Philos. Soc. 29, 297 (1933)

[2] H. Batelaan, Rev. Mod. Phys. 79, 929 (2007).

[3] H. Schwarz, H. A. Tourtellotte, and W. W. Gaertner, Phys. Lett. 19, $202(1965)$.

[4] H. Schwarz, Zeitschrift für Physik 204, 276 (1967)

[5] H. C. Pfeiffer, Phys. Lett. A 26, 362 (1968)

[6] Y. Takeda and I. Matsui, Journal of the Physical Society of Japan 25, 1202 (1968)

[7] L. Bartell, Physics Letters A 27, 236 (1968)

[8] C. Adams, M. Sigel, and J. Mlynek, Phys. Rep. 240, 143 (1994)

[9] M. Freyberger, A. Herkommer, D. Krähmer, E. Mayr, and W. Schleich, in Adv. At., Mol., Opt. Phys. Vol. 41, edited by B. Bederson and H. Walther (Academic Press, New York, 1999) pp. 143-180.

[10] P. L. Gould, G. A. Ruff, and D. E. Pritchard, Phys. Rev. Lett. 56, 827 (1986)

[11] P. J. Martin, B. G. Oldaker, A. H. Miklich, and D. E. Pritchard, Phys. Rev. Lett. 60, 515 (1988)

[12] P. H. Bucksbaum, D. W. Schumacher, and M. Bashkansky, Phys. Rev. Lett. 61, 1182 (1988)

[13] D. L. Freimund, K. Aflatooni, and H. Batelaan, Nature 413, 142 (2001)

[14] D. L. Freimund and H. Batelaan, Phys. Rev. Lett. 89, 283602 (2002)

[15] A. Di Piazza, C. Müller, K. Z. Hatsagortsyan, and C. H. Keitel, Rev. Mod. Phys. 84, 1177 (2012).

[16] V. Yanovsky, V. Chvykov, G. Kalinchenko, P. Rousseau, T. Planchon, T. Matsuoka, A. Maksimchuk, J. Nees, G. Cheriaux, G. Mourou et al., Optics Express 16, 2109 (2008)

[17] F. Amiranoff, F. Augé, H. Backe et al., Proposal for a European Extreme Light Infrastructure, Tech. Rep. (The extreme light infrastructure, 2010) http: //www.extreme-light-infrastructure.eu/pictures/ ELI-scientific-case-id17.pdf

[18] G. Mourou and T. Tajima, Science 331, 41 (2011)

[19] M. Altarelli, R. Brinkmann, M. Chergui, W. Decking, B. Dobson, S. Düsterer, G. Grübel, W. Graeff, H. Graafsma, J. Hajdu et al., eds., The European X-Ray Free-Electron Laser Technical design report (DESY XFEL Project Group European XFEL Project Team Deutsches Elektronen-Synchrotron Member of the Helmholtz Association, Hamburg, 2007).

[20] P. Emma, R. Akre, J. Arthur, R. Bionta, C. Bostedt, J. Bozek, A. Brachmann, P. Bucksbaum, R. Coffee, F.-J. Decker et al., Nature Photon. 4, 641 (2010).

[21] B. W. J. McNeil and N. R. Thompson, Nature Photon. 4, 814 (2010)

[22] M. V. Fedorov, Opt. Commun. 12, 205 (1974)

[23] P. Sancho, Phys. Rev. A 82, 033814 (2010)

[24] P. Sancho, Journal of Physics B: Atomic, Molecular and Optical Physics 44, 145002 (2011)

[25] R. Gush and H. P. Gush, Phys. Rev. D 3, 1712 (1971)

[26] M. A. Efremov and M. V. Fedorov, Journal of Experimental and Theoretical Physics 89, 460 (1999)
[27] M. A. Efremov and M. V. Fedorov, J. Phys. B: At., Mol. Opt. Phys. 33, 4535 (2000)

[28] O. Smirnova, D. L. Freimund, H. Batelaan, and M. Ivanov, Phys. Rev. Lett. 92, 223601 (2004)

[29] V. M. Haroutunian and H. K. Avetissian, Phys. Lett. A 51, 320 (1975)

[30] M. V. Federov and J. K. McIver, Opt. Commun. 32, 179 (1980)

[31] D. L. Freimund and H. Batelaan, Laser Phys. 13, 892 (2003).

[32] L. Rosenberg, Phys. Rev. A 70, 023401 (2004)

[33] P. Krekora, Q. Su, and R. Grobe, J. Phys. B: At., Mol. Opt. Phys. 34, 2795 (2001); M. W. Walser, D. J. Urbach, K. Z. Hatsagortsyan, S. X. Hu, and C. H. Keitel, Phys. Rev. A 65, $043410(2002)$

[34] S. X. Hu and C. H. Keitel, Phys. Rev. Lett. 83, 4709 (1999)

[35] F. H. M. Faisal and S. Bhattacharyya, Phys. Rev. Lett. 93, 053002 (2004)

[36] C. Szymanowski, R. Taïeb, and A. Maquet, Laser Phys. 8, 102 (1998); D. Ivanov, G. Kotkin, and V. Serbo, Eur. Phys. J. C 36, 127 (2004), F. Ehlotzky, K. Krajewska, and J. Z. Kamiński, Rep. Prog. Phys. 72, 046401 (2009)

[37] O. D. Skoromnik, I. D. Feranchuk, and C. H. Keitel, Phys. Rev. A 87, 052107 (2013)

[38] M. Klaiber, E. Yakaboylu, C. Müller, H. Bauke, G. G. Paulus, and K. Z. Hatsagortsyan, (2013), arXiv:1305.5379.

[39] S. Ahrens, H. Bauke, C. H. Keitel, and C. Müller, Phys. Rev. Lett. 109, 043601 (2012)

[40] H. Batelaan, Contemp. Phys. 41, 369 (2000)

[41] B. Thaller, The Dirac Equation, Texts and Monographs in Physics (Springer, Heidelberg, New York, 1992).

[42] L. L. Foldy and S. A. Wouthuysen, Phys. Rev. 78, 29 (1950).

[43] E. de Vries, Fortschritte der Physik 18, 149 (1970)

[44] S. S. Schweber, An introduction to relativistic quantum field theory (Dover, Mineola, 2005).

[45] H. Bauke, S. Ahrens, C. H. Keitel, and R. Grobe, (2013), arXiv:1303.3862

[46] W. H. Press, S. A. Teukolsky, W. T. Vetterling, and B. P. Flannery, Numerical Recipes in $C++($ Cambride University Press, Cambride, 2002).

[47] J. W. Braun, Q. Su, and R. Grobe, Phys. Rev. A 59, 604 (1999)

[48] G. R. Mocken and C. H. Keitel, Comp. Phys. Comm. 178, 868 (2008)

[49] H. Bauke and C. H. Keitel, Comp. Phys. Comm. 182, 2454 (2011)

[50] S. Selst $\varnothing$, E. Lindroth, and J. Bengtsson, Phys. Rev. A 79, 043418 (2009)

[51] F. Fillion-Gourdeau, E. Lorin, and A. D. Bandrauk, Comp. Phys. Comm. 183, 1403 (2012)

[52] C. J. Joachain, N. J. Kylstra, and R. M. Potvliege, Atoms in Intense Laser Fields (Cambridge University Press, Cambridge, 2011).

[53] M. O. Scully and M. S. Zubairy, Quantum Optics (Cambridge University Press, 1997).

[54] S. Ahrens, Investigation of the Kapitza-Dirac effect in the relativistic regime, $\mathrm{Ph} . \mathrm{D}$. thesis, Ruprecht-Karls Universität Heidelberg (2012), http://www.ub.uni-heidelberg.de/ archiv/14049 